\title{
Gravitational-wave detectability of equal-mass black-hole binaries with aligned spins
}

\author{
Christian Reisswig, ${ }^{1}$ Sascha Husa ${ }^{2,1}$ Luciano Rezzolla, ${ }^{1,3}$ Ernst Nils Dorband, ${ }^{1}$ Denis Pollney, ${ }^{1}$ and Jennifer Seiler ${ }^{1}$ \\ ${ }^{1}$ Max-Planck-Institut für Gravitationsphysik, Albert-Einstein-Institut, Potsdam-Golm, Germany \\ ${ }^{2}$ Departament de Física, Universitat de les Illes Balears, Palma de Mallorca, Spain \\ ${ }^{3}$ Department of Physics and Astronomy, Louisiana State University, Baton Rouge, LA, USA
}

(Dated: October 24, 2018)

\begin{abstract}
Binary black-hole systems with spins aligned or anti-aligned to the orbital angular momentum, and which therefore do not exhibit precession effects, provide the natural ground to start detailed studies of the influence of strong-field spin effects on gravitational wave observations of coalescing binaries. Furthermore, such systems may be the preferred end-state of the inspiral of generic supermassive binary black-hole systems. In view of this, we have computed the inspiral and merger of a large set of binary systems of equal-mass black holes with spins parallel to the orbital angular momentum but otherwise arbitrary. Our attention is particularly focused on the gravitational-wave emission so as to quantify how much spin effects contribute to the signal-to-noise ratio, to the horizon distances, and to the relative event rates for the representative ranges in masses and detectors. As expected, the signal-to-noise ratio increases with the projection of the total black hole spin in the direction of the orbital momentum. We find that equal-spin binaries with maximum spin aligned with the orbital angular momentum are more than "three times as loud" as the corresponding binaries with anti-aligned spins, thus corresponding to event rates up to 30 times larger. We also consider the waveform mismatch between the different spinning configurations and find that, within our numerical accuracy, binaries with opposite spins $\boldsymbol{S}_{1}=-\boldsymbol{S}_{2}$ cannot be distinguished whereas binaries with spin $\boldsymbol{S}_{1}=\boldsymbol{S}_{2}$ have clearly distinct gravitationalwave emissions. Finally, we derive a simple expression for the energy radiated in gravitational waves and find that the binaries always have efficiencies $E_{\mathrm{rad}} / M \gtrsim 3.6 \%$, which can become as large as $E_{\mathrm{rad}} / M \simeq 10 \%$ for maximally spinning binaries with spins aligned with the orbital angular momentum. These binaries are therefore among the most efficient sources of energy in the Universe.
\end{abstract}

PACS numbers: 04.25.Dm, 04.30.Db, 95.30.Sf, 97.60.Lf

\section{INTRODUCTION}

It has been a long-standing goal of the field of numerical relativity to provide results for gravitational-wave data analysis and thus enhance the capabilities of current and future gravitational wave detectors, in particular regarding the observation of compact binary coalescence. With a series of breakthroughs in 2005 [1-3], this long-term goal has suddenly become reality. However, much further work is required to actually understand the practical implications of numerical solutions of the full Einstein equations for gravitational-wave data analysis. Indeed, first studies suggest that template banks that use numerical information can increase the reach of detectors [4-6], aid the calibration of search pipelines [7-9], and improve the estimation of parameters, such as e.g., sky location [10].

In this paper we use gravitational waveforms from numerical-relativity (NR) calculations of a number of sequences of equal-mass spinning black-hole binaries whose spins are aligned (anti-aligned) with the orbital angular momentum, and consider the detectability of these binaries for the ground-based gravitational wave-detectors as well as for the planned space-based LISA interferometer.

Our interest in this type of binary stems from the fact that there are indications they represent preferred configurations in nature, at least if the black holes are supermassive. It has been shown, in fact, that when the binary is surrounded by a massive circumbinary disc, as the one expected by the merger of two galaxies, the dissipative dynamics of the matter produces a torque with the effect of aligning the spins to the orbital angular momentum [11]. In addition, the merger of binaries with aligned spins yields recoil velocities which are sufficiently small (i.e., $\lesssim 450 \mathrm{~km} / \mathrm{s}[12-14])$ to prevent the final black hole from being expelled from the host galaxy. This would then be compatible with the overwhelming astronomical evidence that massive black holes reside at the centers of most galaxies.

Our parameter space is therefore 2-dimensional, parametrized by the projections $a_{1}, a_{2}$ of the dimensionless spins $\boldsymbol{a}_{i} \equiv \boldsymbol{S}_{i} / M_{i}^{2}$ of the individual black holes on to direction of the angular momentum (chosen as the $z$-axis). As a result, spins that are aligned with the orbital angular momentum are characterized by positive values of $a_{1}$, $a_{2}$, while anti-aligned spins have negative values. Previous studies of this parameter space [12-26], have considered the recoil velocity and final spin of the merger remnant, and have constructed phenomenological formulas for these quantities given the initial spins $a_{1}$ and $a_{2}$ of the binary.

In this work, we move our focus to the detectability of a given set of binaries in the parameter sub-space of (anti-) aligned spins, i.e., for each of these binaries and across a set of different masses we calculate the signal-to-noise ratio (SNR) for the LIGO [27, 28], enhanced LIGO (eLIGO) [29], advanced LIGO (AdLIGO) [5, 30], Virgo [31], advanced Virgo (AdVirgo) [32], and LISA [33, 34] detectors .

In this way we attempt to address the following questions:

(i) Which among the aligned-spin configurations is the "loudest" and which one is the "quietest"?

(ii) How large is the difference in signal-to-noise ratio between the loudest and the quietest? 
(iii) How do these considerations depend on the detector used, the mass of the binary, and the number of harmonics?

(iv) Are there configurations whose waveforms are difficult to distinguish and are hence degenerate in the space of templates?

Overall, and as expected, we find that equal-spinning, maximally anti-aligned binaries generally produce the lowest SNR while equal-spinning, maximally aligned binaries produce the highest SNR. For any mass, the SNR can be well described with a low-order polynomial of the initial spins $\rho=\rho\left(a_{1}, a_{2}\right)$ and generally it increases with the total dimensionless spin along the angular momentum direction, $a \equiv \frac{1}{2}\left(\boldsymbol{a}_{1}+\boldsymbol{a}_{2}\right) \cdot \hat{\boldsymbol{L}}$. The possibility of describing the whole behaviour of the waveforms from equal-mass, aligned/antialigned binaries in terms of a single scalar quantity, namely $a$, provides a certain amount of optimism that also more complex spin configurations can, ultimately, be described in terms of a few parameters only.

We also analyze the impact that modes of the gravitationalwave field of order larger than $\ell=2$ but smaller than $\ell=5$ have on the maximum SNR and show that for low masses $M \in[20,100]$ they contribute, say for the LIGO detector, $\approx 2.5 \%$, whereas for intermediate masses $M>100 M_{\odot}$ they contribute $\approx 8 \%{ }^{1}$. In addition, we determine the ratio between maximum and averaged SNR for $\ell>2$ which is known to be $\sqrt{5}$ when considering only the $\ell=2, m=2$ mode. We also calculate the mismatch between the waveforms from different binaries across our spin-diagram and find that binaries along the diagonal $a_{1}=-a_{2}$ cannot be distinguished within our given numerical accuracy, whereas configurations along the diagonal $a_{1}=a_{2}$ are clearly different ( $c f$. Fig 7 and 8 , as well as Table IV. Finally, we derive a simple expression for the energy radiated in gravitational waves and find that this is bounded between $\simeq 3.6 \%$ and $\simeq 10 \%$ for maximally spinning binaries with spins anti-aligned or aligned with the orbital angular momentum, respectively.

The plan of the paper is as follows: in Sect. II we recall very briefly the numerical set up and illustrate the properties of the initial data used in the simulations. Sect.III is dedicated to the discussion of the gravitational-wave observables used for the subsequent analysis, while Sect.IV presents the results in terms of the SNR and how this is influenced by higher-order modes. This Section also contains a discussion of the match between the waveforms from different binaries and an assessment of the accuracy of our results. Sect. $\mathrm{V}$, on the other hand, provides a brief discussion of the analytic expressions we have found representing either the SNR or the energy radiated in gravitational waves. Finally, conclusions are summarized in Sect.VI.

\footnotetext{
${ }^{1}$ Note that for some specific angles at which the SNR is not maximum, the contribution of the higher modes can be much more significant
}

\section{NUMERICAL SETUP AND INITIAL DATA}

The numerical simulations have been carried out using the CCATIE code, a three-dimensional finite-differencing code solving a conformal-traceless " $3+1$ " BSSNOK formulation of the Einstein equations [15] using the Cactus Computational Toolkit [35] and the Carpet [36] adaptive mesh-refinement driver. The main features of the code have been presented in several papers, and recently reviewed in Pollney et al. [15]. The code implements the "moving-punctures" technique to represent dynamical black holes following [2, 37] (see also [38, 39]), which has proven to be a robust way to evolve blackhole spacetimes.

For compactness we will not report here the details of the formulation of the Einstein equations solved or the form of the gauge conditions adopted. All of these aspects are discussed in great detail in [15], to which we refer the interested reader. More specific to these simulations, however, is the numerical grid setup. In the results presented below we have used 9 levels of mesh refinement with a fine-grid resolution of $\Delta x / M=0.02$ and fourth-order finite differencing. The wave-zone grid has a resolution of $\Delta x / M=0.128$ and extends from $r=24 M$ to $r=180 \mathrm{M}$, in which our wave extraction is carried out. The outer (coarsest) grid extends to a spatial position which is $819.2 M$ in each coordinate direction. Furthermore, because the black holes spins are all directed along the $z$-axis of our Cartesian grids, it is possible to use a reflection symmetry condition across the $z=0$ plane.

The initial data are constructed applying the "puncture" method [40-43] as described in [44]. We have considered four different sequences labelled as “ $r$ ", “ $s$," “ $t$ ”, and “ $u$ ” along straight lines in the $\left(a_{1}, a_{2}\right)$ parameter space, also referred to as the "spin diagram" ( $c f$. Table $\square$ for details). As shown in Fig. 1 these sequences allow us to cover the most important portions of the space of parameters which, we recall, is symmetric with respect to the $a_{1}=a_{2}$ diagonal.

We note that similar sequences have also been considered in [12, 15- 18] but have here been recalculated both using a higher resolution and with improved initial orbital parameters. More specifically, we use post-Newtonian (PN) evolutions following the scheme outlined in [45], which provides a straightforward prescription for initial-data parameters with small initial eccentricity, and which can be interpreted as part of the process of matching our numerical calculations to the inspiral described by the PN approximations. The free parameters to be chosen for the puncture initial data are therefore: the puncture coordinate locations $\boldsymbol{C}_{i}$, the puncture bare mass parameters $m_{i}$, the linear momenta $\boldsymbol{p}_{i}$, and the individual spins $\boldsymbol{S}_{i}$. The initial parameters for all of the binaries considered are collected in the left part of Table \. The initial separations are fixed at $D=8 M$, where $M$ is the total initial black hole mass, chosen as $M=1$ (note that the initial ADM mass of the spacetime is not exactly 1 due to the binding energy of the black holes), while the individual asymptotic initial black hole masses are therefore $M_{i}=1 / 2$. The only exception is for the binary $s_{-8}$, for which $D=10 \mathrm{M}$. 
TABLE I: Binary sequences for which numerical simulations have been carried out, with various columns referring to the puncture initial location $\pm x / M$, the mass parameters $m_{i} / M$, the dimensionless spins $a_{i}$, and the normalized ADM mass $\widetilde{M}_{\mathrm{ADM}} \equiv M_{\mathrm{ADM}} / M$ measured at infinity. Finally, the last four columns contain the numerical values of the energy radiated during the simulation using the two methods described in the text and the corresponding errors between them, as well as the error to the fitted values.

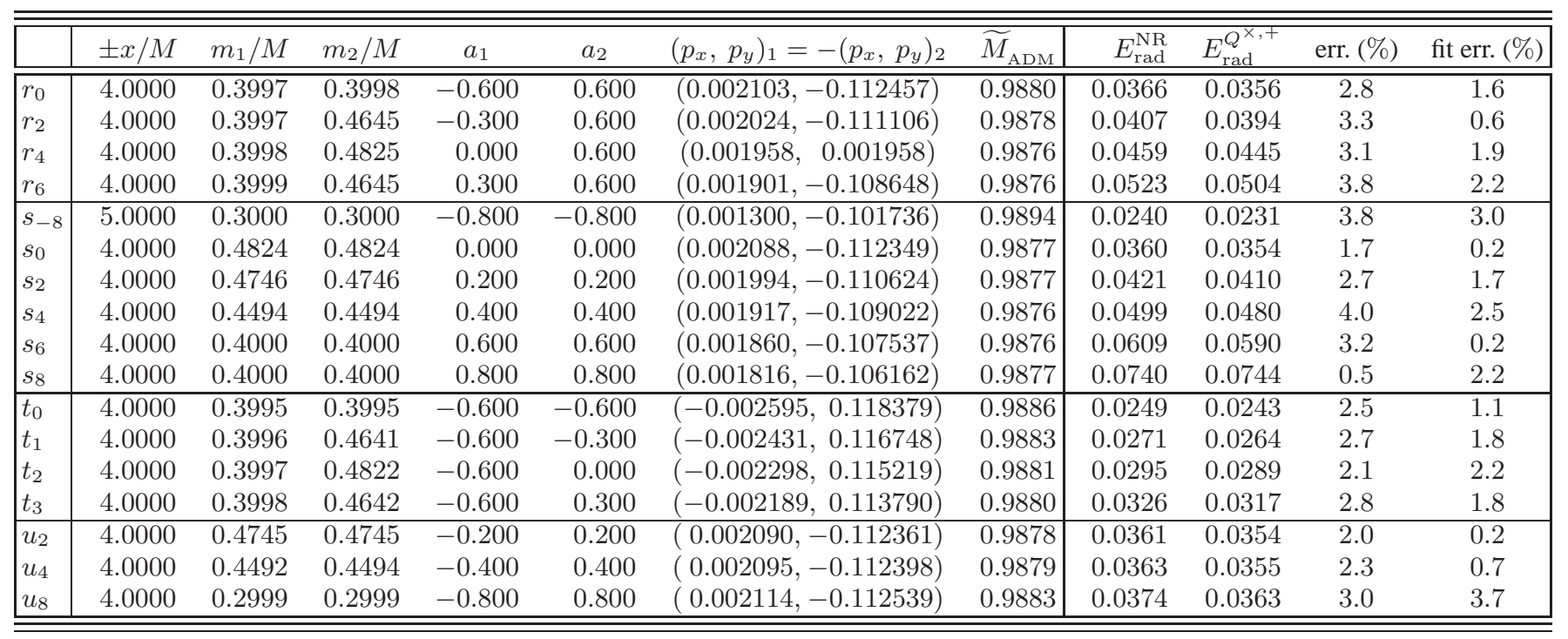

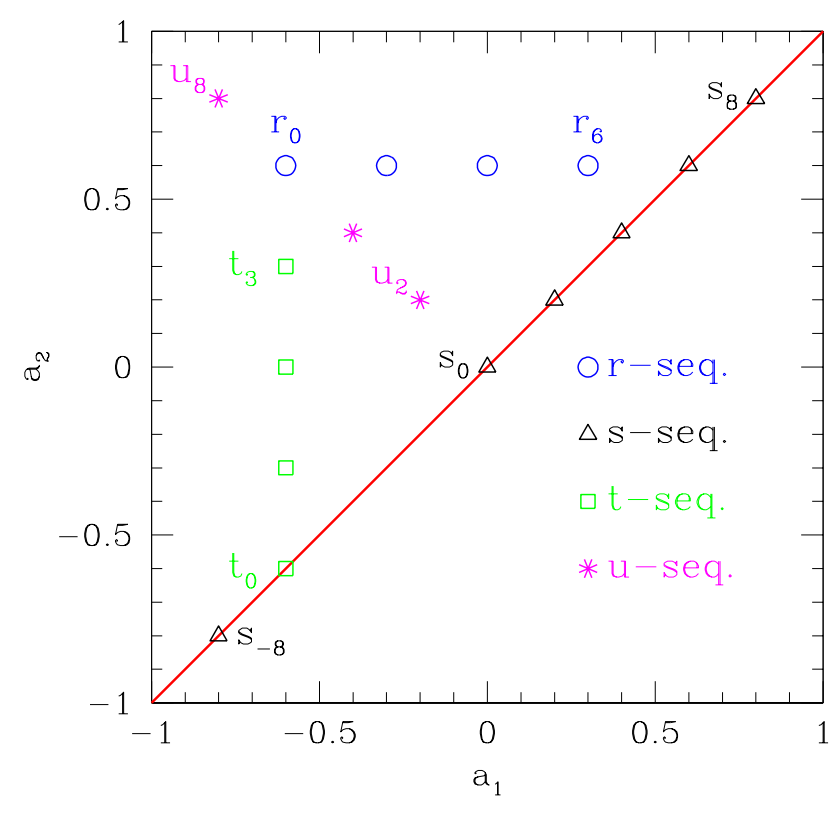

FIG. 1: Schematic representation in the $\left(a_{1}, a_{2}\right)$ plane, also referred to as the "spin diagram", of the initial data collected in Table凹 These sequences cover most important portions of the space of parameters which is symmetric with respect to the $a_{1}=a_{2}$ diagonal.

\section{GRAVITATIONAL-WAVE OBSERVABLES}

In this Section we discuss the gravitational-wave observables that have been studied from the sample reported in Ta- ble $\prod$ and how these have been used to compute the radiated energy, the SNR, the horizon distances and the event rates.

\section{A. Numerical-Relativity (NR) waveforms}

Although the CCATIE code computes the gravitational waveforms either via the Newman-Penrose curvature scalar $\Psi_{4}$ or via gauge-invariant metric perturbations on a Schwarzschild background, the analysis carried hereafter will be made in terms of the latter. While the two prescriptions yield, in fact, estimates which are in very good agreement with each other and with differences below $2 \%$ (see discussion in [15]), we have found that the results obtained using gauge-invariant quantities have a smaller numerical error, and are thus preferable.

More specifically, we compute the gravitational-wave amplitudes $h_{\ell m}^{+}$and $h_{\ell m}^{\times}$in terms of the even and odd master functions $Q_{\ell m}^{+}$and $Q_{\ell m}^{\times}$via the relations [46]

$h_{\ell m}(t)=h_{\ell m}^{+}(t)-\mathrm{i} h_{\ell m}^{\times}(t)=Q_{\ell m}^{+}(t)-\mathrm{i} \int_{-\infty}^{t} d t^{\prime} Q_{\ell m}^{\times}\left(t^{\prime}\right)$,

where the gauge-invariant perturbations are typically extracted at a radius of $r_{\mathrm{E}}=160 M$ (see Sec. IVD for a discussion of the accuracy of our measurements and ref. [15] for a comparison among different extraction radii).

As mentioned before, all our binaries [but $s_{-8}$ ] have initial separations of $D=8.0 M[D=10.0 M]$, which, in the parameter space that we have considered, leads to a maximum initial frequency of the numerical waveforms, that is $\omega_{\text {ini }}=0.084 / M$. Depending therefore on the mass $M$, such an initial frequency can be greater than the lower cut-off frequency of the detector $\omega_{\text {co }}$ for a given source at an arbitrary 
TABLE II: Initial instantaneous frequencies $M \omega_{\text {ini }}$ and associated minimum masses $M_{\min }$ of the NR waveforms for the different models and for each detector according to the corresponding lower cut-off frequency (i.e., at $30 \mathrm{~Hz}$ for Virgo, at $40 \mathrm{~Hz}$ for eLIGO, at $10 \mathrm{~Hz}$ for AdLIGO/AdVirgo, and at $10^{-4} \mathrm{~Hz}$ for LISA). All the values for the masses are in units of solar masses.

\begin{tabular}{|l|c|cccc|}
\hline \hline \hline & $M \omega_{\text {ini }}$ & $\begin{array}{c}M_{\min } \\
\text { Virgo }\end{array}$ & $\begin{array}{c}M_{\min } \\
\text { eLIGO }\end{array}$ & $\begin{array}{c}M_{\min } \\
\text { AdLIGO/AdVirgo }\end{array}$ & $\begin{array}{c}M_{\min } \\
\text { LISA }\end{array}$ \\
\hline \hline$r_{0}$ & 0.080 & 86.2 & 64.6 & 258.5 & $2.58 \times 10^{7}$ \\
$r_{2}$ & 0.078 & 84.0 & 63.0 & 252.0 & $2.52 \times 10^{7}$ \\
$r_{4}$ & 0.077 & 82.9 & 62.2 & 248.8 & $2.49 \times 10^{7}$ \\
$r_{6}$ & 0.076 & 81.8 & 61.4 & 245.5 & $2.46 \times 10^{7}$ \\
\hline$s_{-8}$ & 0.060 & 64.6 & 48.4 & 193.8 & $1.93 \times 10^{7}$ \\
$s_{0}$ & 0.080 & 86.2 & 64.6 & 258.5 & $2.58 \times 10^{7}$ \\
$s_{2}$ & 0.078 & 84.0 & 63.0 & 252.0 & $2.52 \times 10^{7}$ \\
$s_{4}$ & 0.076 & 81.8 & 61.4 & 245.5 & $2.46 \times 10^{7}$ \\
$s_{6}$ & 0.075 & 80.8 & 60.6 & 242.3 & $2.42 \times 10^{7}$ \\
$s_{8}$ & 0.073 & 78.6 & 59.0 & 235.8 & $2.36 \times 10^{7}$ \\
\hline$t_{0}$ & 0.084 & 90.5 & 67.8 & 271.4 & $2.71 \times 10^{7}$ \\
$t_{1}$ & 0.083 & 89.4 & 67.0 & 268.2 & $2.68 \times 10^{7}$ \\
$t_{2}$ & 0.082 & 88.3 & 66.2 & 264.9 & $2.65 \times 10^{7}$ \\
$t_{3}$ & 0.081 & 87.2 & 65.4 & 261.7 & $2.62 \times 10^{7}$ \\
\hline$u_{2}$ & 0.080 & 86.2 & 64.6 & 258.5 & $2.58 \times 10^{7}$ \\
$u_{4}$ & 0.080 & 86.2 & 64.6 & 258.5 & $2.58 \times 10^{7}$ \\
$u_{8}$ & 0.080 & 86.2 & 64.6 & 258.5 & $2.58 \times 10^{7}$ \\
\hline \hline
\end{tabular}

distance. Because we expect that for most masses $\omega_{\text {co }}$ will be smaller than $\omega_{\text {ini }}$, we need to provide additional information about the gravitational-wave signal in the frequency range between $\omega_{\text {co }}$ and $\omega_{\text {ini }}$. This can be accomplished by "gluing" the NR waveform with a PN part as discussed in the next Section.

The values of the initial frequencies and of the associated minimum masses $M_{\min }$ for each of the detectors considered are reported in Table

\section{B. Matching PN and NR waveform amplitudes}

The existence of a cut-off mass set by the initial frequency of the NR simulations would clearly restrict the validity of our considerations to large masses only. To counter this and thus include also binaries with smaller masses, we account for the early inspiral phase by describing it via PN approximations. To produce the PN waveforms, and the PN energy that we are using directly in Sec. VB, we have used the spinning TaylorT1 approximant used in Hannam et al. [47], and which is based on the PN expressions described in [48-55]. The choice of TaylorT1 is motivated by that fact, that in [47] it is found to be more robust in the spinning case than the TaylorT4 approximant, which was previously found to yield excellent results in the nonspinning case [56] (see e.g., [56] for a comparison of different techniques to obtain the gravitational-wave phase information for quasi-circular inspiral). These waveforms are 3.5 PN accurate in the nonspinning phase, and 2.5 PN accurate in the spin-dependent terms entering the phasing. The gravitational-wave amplitudes, on the other hand, have been computed according to ref. [57] (see also [58]) to the high- est PN order that is currently known for each of the spherical harmonic modes that we use.

A phase-coherent construction of hybrid PN-NR waveforms is rather delicate, and has not yet been achieved for the higher spherical harmonic modes we use here (see [4, 5] for some recent work in the case of nonspinning binaries). However, for the present purpose of computing the SNR and the radiated energies, such a construction in the time domain is not necessary and all of the relevant work can be done much more simply in the frequency domain. In practice, we Fourier transform the PN and NR waveforms and "glue" them together

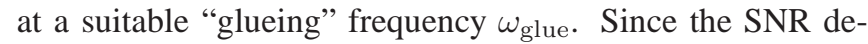
pends only on the amplitude of the waveform, [cf. eq. (5)], it is not necessary to match the PN-waveform in the phase. This greatly simplifies the process of waveform matching and basically reduces to a simple check of the amplitude matching to address the error of the mismatch. Indeed, we have found that without any parameter adjustment, the PN-waveform amplitudes match rather well with the inspiral part of the NRwaveforms, and result in an error which is usually $\approx 1.5 \%$ and in the worst case $\approx 4.0 \%$ for the binary configuration $t_{0}$. The only care which is important to pay in the timedomain analysis, and in order to limit the noise artifacts in the Fourier-transformed amplitudes, is the use of a windowing function (e.g., a hyperbolic tangent) to smoothly blend the waveform to zero before the initial burst of spurious radiation and after the ringdown, in order to limit spurious oscillations in the Fourier-transformed waveform. A representative example is shown in Fig. 2, where we report the noise strain for the Virgo and Advanced LIGO detectors, together with the Fourier-transformed amplitude of the PN and NR waveform for the maximally spinning model $s_{8}$. The waveform is assumed to be observed at $\theta=0, \phi=0$ for a total mass $M=200 M_{\odot}$ and from a distance $d=100 \mathrm{Mpc}$. The glueing frequency in this case is at $f_{\text {glue }}=\omega_{\text {glue }} /(2 \pi)=27.14$ $\mathrm{Hz}$.

Since each $\ell, m$ mode of the gravitational-wave field will have a different initial frequency, we need to make sure that they are all properly taken into account when determining the glueing frequency, so that, at least in principle

$$
\omega_{\text {glue }} \geq \max _{\ell, m}\left(\omega_{\text {ini }}\right)_{\ell m}
$$

In practice, the initial frequency of our highest mode, $\ell=$ $4, m=4$, has an initial frequency $\left(\omega_{\text {ini }}\right)_{44}=2\left(\omega_{\text {ini }}\right)_{22}$. As a result, we select the glueing frequency according to the binary configuration with the largest initial frequency, i.e., the binary $t_{0}$, and take $\omega_{\text {glue }}=2\left(\omega_{\text {ini }}\right)_{22}=0.168 / M$. We also measure how sensitive this choice is, by considering how the results are affected when choosing instead $\omega_{\text {glue }} \pm \Delta \omega$, with $\Delta \omega \ll \omega_{\text {glue }}$. More specifically, for $\Delta \omega=0.01 / M$ we find a maximal difference in the computed SNR of $\sim 2.0 \%$ over all configurations and all masses. Note that such a difference affects equally the maximum and averaged SNRs (see Sect. IIID for a discussion on these two different measures of the SNR). Furthermore, a change of $\Delta \omega$ in $\omega_{\text {glue }}$ affects only marginally the relative difference between SNRs computed by including modes up to $\ell=2$ and $\ell=4$, and also in this case 


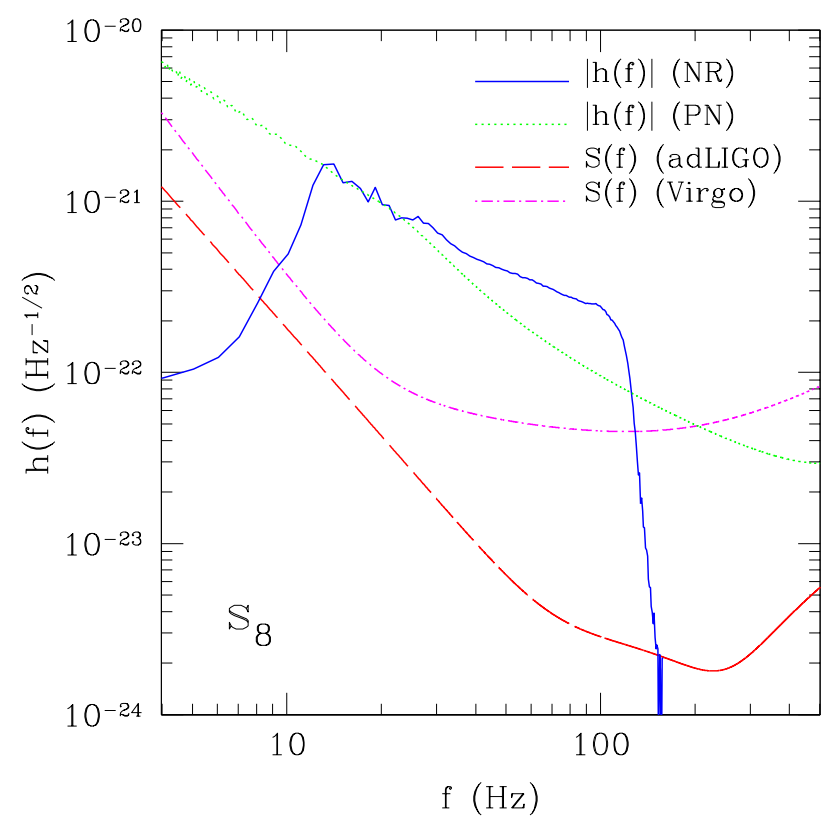

FIG. 2: Noise strain for the Advanced LIGO and Virgo detectors and the Fourier-transformed amplitude of the PN and NR waveform at $\theta=0, \phi=0$ for a total mass $M=200 M_{\odot}$ at a distance $d=100 \mathrm{Mpc}$ for the maximally spinning model $s_{8}$. The glueing frequency is at $f_{\text {glue }}=27.14 \mathrm{~Hz}$.

the differences are $\sim 2.0 \%$. Overall, therefore, the uncertainties introduced by the choice of $\omega_{\text {glue }}$ are much smaller than the typical error at which we report the SNRs.

\section{Radiated Energy}

Since the total energy must be conserved, we can use the radiated energy as an important tool to verify the accuracy of the gravitational-wave amplitude and thus the overall precision of our calculations. More specifically, because it is straightforward to determine the initial and the final total mass, it is also straightforward to compare the difference in the two with the radiated energy. In practice, we compute the initial mass of the system as $M_{\mathrm{ini}}=\widetilde{M}_{\mathrm{ADM}}$, while the final mass of the merger remnant $M_{\text {fin }}$ is deduced from the properties of the apparent horizon within the isolated-horizon formalism as first discussed in [59] and then extensively investigated in [60]. The radiated energy is then simply given by the difference

$$
E_{\mathrm{rad}}^{\mathrm{NR}}=M_{\mathrm{ADM}}-M_{\mathrm{fin}},
$$

and should be equal to the energy that has been radiated through gravitational waves during the simulation [46]

$$
E_{\mathrm{rad}}^{Q^{\times,+}}=\frac{1}{32 \pi} \sum_{\ell, m} \int_{0}^{t} d t^{\prime}\left(\left|\frac{d Q_{\ell m}^{+}}{d t}\right|^{2}+\left|Q_{\ell m}^{\times}\right|^{2}\right) .
$$

Overall, we have found that for all binaries the difference between $E_{\mathrm{rad}}$ and $E_{\mathrm{rad}}^{Q^{\times,+}}$is between $\sim 0.5 \%$ and $\sim 4.0 \%$ and a detailed comparison of the numerical values is reported in Table I In Sect. VB we will discuss an analytic fit to the computed data that provides a simple-to-use measure of the amount of mass radiated during the inspiral, merger and ringdown as a function of the initial spins.

\section{SNR, Horizon Distances and Event Rates}

Following ref. [61], we define the SNR, $\rho$, for matchedfiltering searches as

$$
\rho^{2} \equiv\left(\frac{S}{N}\right)_{\text {matched }}^{2}=4 \int_{0}^{\infty} \frac{|\tilde{h}(f)|^{2}}{S_{h}(f)} d f,
$$

where $\tilde{h}(f)$ is the Fourier transform of the time domain gravitational-wave signal $h(t)$, defined in the continuum as

$$
\tilde{h}(f)=\int_{-\infty}^{\infty} h(t) e^{-2 \pi \mathrm{i} f t} d t
$$

and $S_{h}(f)$ is the noise power spectral density for a given detector. Hereafter we will consider the $S_{h}(f)$ for the groundbased detectors LIGO, enhanced LIGO, advanced LIGO and Virgo, as well as the space-bound LISA interferometer. [The associated noise power spectral densities are reported in Appendix A]

Note that since the SNR (5) depends on the angle from the source to the detector, it is useful to introduce the angleaveraged SNR $\left\langle\rho^{2}\right\rangle$, which can be computed straightforwardly after decomposing the gravitational-wave signal in terms of spherical harmonic modes. More specifically, using the orthonormality of the spin-weighted spherical harmonic basis ${ }_{s} Y_{\ell m}$, the "angle-averaged" SNR

$$
\rho_{\text {avg }} \equiv\left\langle\rho^{2}\right\rangle \equiv \frac{1}{\pi} \int d \Omega \int d f \frac{\left|\sum_{\ell m} \tilde{h}_{\ell m}(f)-2 Y_{\ell m}(\Omega)\right|^{2}}{S_{h}(f)}
$$

can be written as a simple sum of integrals of the absolute squares of the Fourier-transformed modes $\tilde{h}_{\ell m}(f)$

$$
\rho_{\mathrm{avg}}=\frac{1}{\pi} \sum_{\ell m} \int d f \frac{\left|\tilde{h}_{\ell m}(f)\right|^{2}}{S_{h}(f)}
$$

and hence it can be evaluated straightforwardly. For each binary, distance and mass, we have calculated both the "maximum" SNR $\rho_{\max }$ for an optimally oriented detector, i.e., the SNR for a detector oriented such that it measures only the + polarization of the gravitational-wave signal, and the averaged SNR. Here the mass is always meant to be the redshifted total mass, i.e., $(1+z) M_{\text {source, }}$, where $z$ is the redshift and $M_{\text {source }}$ is the mass at the source. For sources at small distances, i.e., less than $100 \mathrm{Mpc}$, then $z \lesssim 0.024$ and hence $M \simeq M_{\text {source }}$ to within a few percent. Identical results would have been obtained if we had considered the $\times$ polarization.

It is worth noting that if the gravitational-wave signal is modeled simply through the dominant $\ell=2=m$ mode (or 
in our case via a superposition $\ell=2= \pm m)^{2}$, the maximum SNR can be deduced from the average SNR after exploiting the properties of the spin-weighted spherical harmonic ${ }_{-2} Y_{22}$ and ${ }_{-2} Y_{2-2}$, namely,

$$
\begin{aligned}
\rho_{\max } & =\sqrt{5 \rho_{\mathrm{avg}}^{2}(\ell=2, m=2)} \\
& =\sqrt{\frac{5}{2} \rho_{\mathrm{avg}}^{2}(\ell=2, m= \pm 2)} .
\end{aligned}
$$

However, such a relation is no longer true when including modes with $\ell>2$, and the relation between the maximum and the averaged value of the SNR can only be determined numerically.

When computing the SNR, a reference distance needs to be fixed and we have set such a distance to be $d_{\rho}=100 \mathrm{Mpc}$. The results of the SNR at $d_{\rho}$ across the spin diagram can then be recast in terms of an "horizon distance", namely the distance at which a given binary system with redshifted mass $M$ has an SNR equal to a threshold for detectability, which we chose to be $\rho=8$, as customary for ground-based detectors. The horizon distance is then simply defined as

$$
d_{H}=d_{\rho}\left(\frac{\rho\left(d=d_{\rho}\right)}{8}\right) \mathrm{Mpc} .
$$

The quantity $d_{H}$ is clearly equivalent to the SNR but has the advantage to provide, at least for detectors not operating at large SNRs, a simple estimate of the increase in the relative event rate $R$ as

$$
R \sim\left(\frac{d_{H}}{d_{H, a=-1}}\right)^{3}
$$

where $d_{H, a=-1}$ is the horizon distance of the configuration with lowest SNR, i.e., which belongs to the extrapolated case $a=-1$. Although simple, this formula requires a caveat. Expression (12) is valid as an equality only for small horizon distances, namely those for which the redshift is negligible. This is because at large redshifts the observed masses would differ considerably from the masses at the source. In other words, at large redshifts the horizon distances would be different not only because of the spin, but also because the masses at the sources would be intrinsically different. This clearly impacts the deduced event rate as defined in (12), which considers only the contributions coming from the spin. Hence, for large redshifts the event rate $R$ defined here serves only as a lower limit for masses larger than the optimal one and, vice versa, as an upper bound for masses smaller than the optimal.

To fix the ideas, let us consider a concrete example. Let us assume that we have calculated the horizon distance for a binary with $a=-1$ which, as can be deduced from Fig. 4 and will be discussed in the next Section, will lead to the smallest SNR for a given detector. We also assume that this binary

\footnotetext{
${ }^{2}$ Note that in our binary configurations due to symmetry, we always have $h_{\ell m}=h_{\ell-m}$
}

has a mass at the detector which is smaller than the optimal one. Let us now consider a binary with the same mass at the detector but with $a>-1$; this binary will clearly lead to a larger SNR but because the masses at the detector are the same, the mass of the binary with $a>-1$ will be (because of the redshift) smaller at the source. As a result, its horizon distance will be overestimated, and hence the event rate coming from (12) only an upper bound. A similar argument for masses larger than the optimal one would instead lead to the conclusion that the event rate $R$ is only a lower bound.

\section{RESULTS}

In what follows we discuss the results obtained in terms of the SNR and how this is influenced by higher-order modes. We also discuss the match between the waveforms from different binaries and an assessment of the accuracy of our results.

\section{A. Horizon distances and SNRs}

The results of the analysis discussed above are nicely summarized in Fig. 3, which shows the averaged and maximum horizon distance $d_{H}=d_{H}(a, M)$ for some of the detectors considered. As mentioned above, the horizon distance has been computed at a reference $\operatorname{SNR} \rho=8.0$, and is parametrized in terms of the total mass of the system (in solar masses) and of the average dimensionless spin " $a$ " as projected along the orbital angular momentum $\boldsymbol{L}$

$$
a \equiv \frac{1}{2}\left(\boldsymbol{a}_{1}+\boldsymbol{a}_{2}\right) \cdot \hat{\boldsymbol{L}}=\frac{1}{2}\left(\boldsymbol{a}_{1}+\boldsymbol{a}_{2}\right) \cdot \boldsymbol{e}_{z},
$$

where $\hat{\boldsymbol{L}} \equiv \boldsymbol{L} /|\boldsymbol{L}|$, and the orbital plane has been chosen to coincide with the $(x, y)$ plane of our Cartesian coordinate system. More specifically, the top left panel of Fig. 3 3 refers to the LIGO detector, the top right panel to the Virgo detector, while the lower left and right panels refer to the advanced versions of both detectors, respectively.

While quite self-explanatory, these panels deserve some comments. First, as expected, the maximum SNR is always larger than the average one but the difference between the two is not constant, changing both with the total dimensionless spin $a$ and with the total mass $M$. Second, for any fixed value of $a$, the horizon distance (and hence the SNR) grows steeply to a maximum mass and then rapidly decreases to very small values of $\sim \mathcal{O}(1)$. Clearly, this reflects the existence of a sweet-spot in the sensitivity curve of all detectors. Third, for any value of $a$, the maximum horizon distance/SNR also marks the "optimal mass" for the binary $M_{\mathrm{opt}}$, namely the mass of the binary whose inspiral and merger is optimally tuned with the given detector and hence can be seen from further away. Note that the differences between the maximum and average SNR are largest in the neighborhood of the optimal mass. Fourth, the configuration with spins parallel and aligned to the orbital angular momentum are generically "louder" than those with spins parallel but antialigned with the 

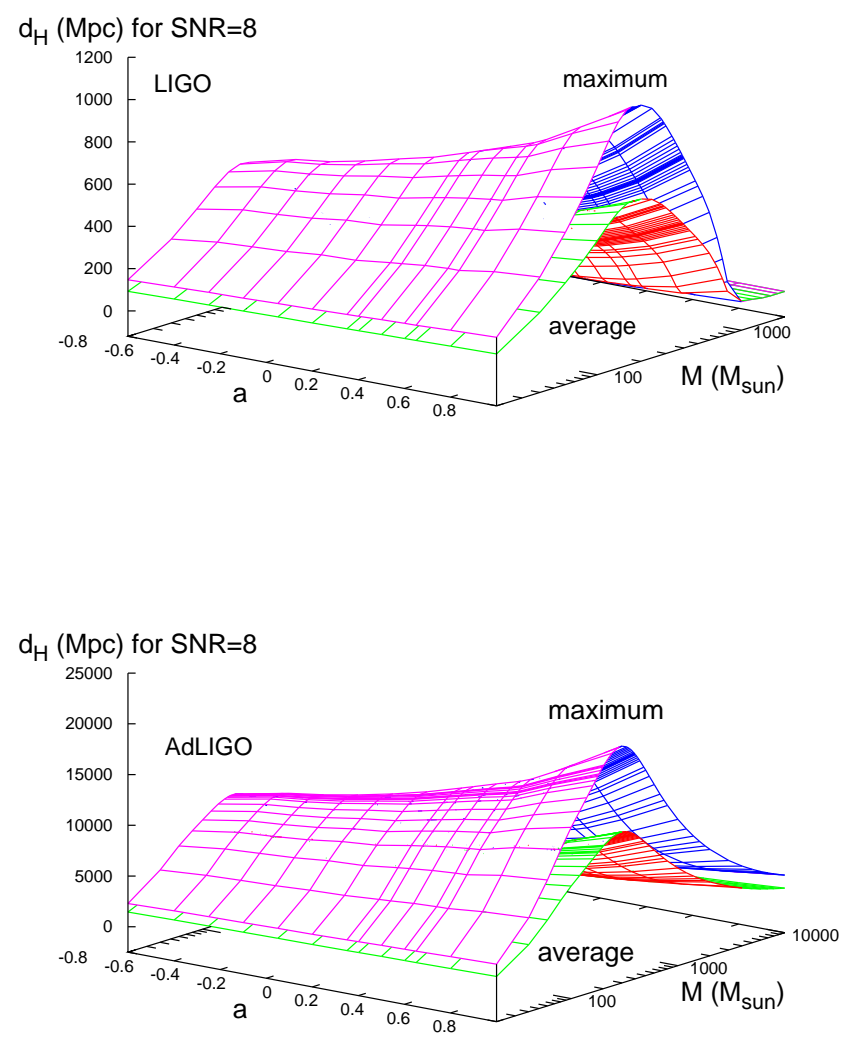
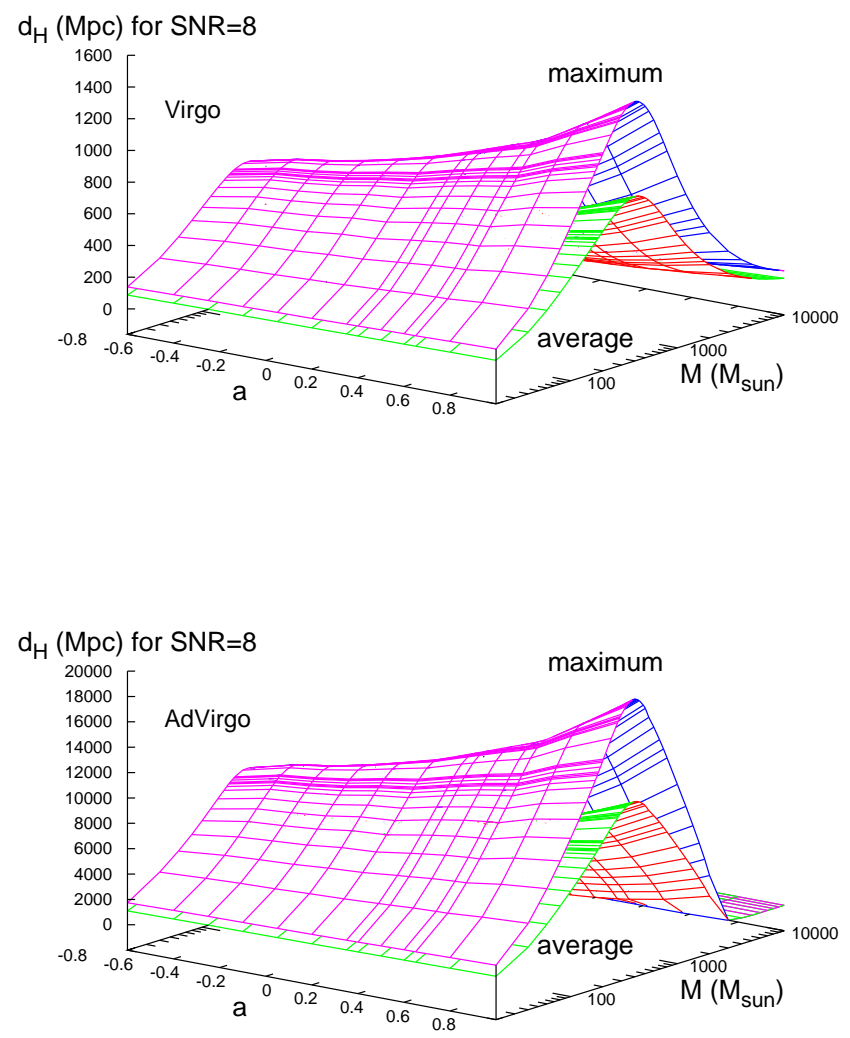

FIG. 3: Averaged and maximum horizon distance $d_{H}=d_{H}(a, M)$ for the LIGO detector (top left panel), for the Virgo detector (top right panel), and for the advanced versions of both detectors (bottom left and right panels, respectively). The horizon distance has been computed at a reference $\operatorname{SNR} \rho=8.0$.

orbital angular momentum, with the binaries having $a= \pm 1$ being the "loudest" and "quietest", respectively; this is essentially the answer to question $(i)$ in the Introduction. ${ }^{3}$. Fifth, in the cases of the LIGO and advanced Virgo detectors the horizon distance is essentially zero at cut-off masses which are $\sim 900 M_{\odot}$ and $\sim 3000 M_{\odot}$, respectively. Sixth, for any fixed value of the total mass, the SNR grows with $a$ and, as we will discuss later on, this growth is very well described with a polynomial of 4 th order ( $c f$. discussion in Sect. VA). This is shown more clearly in Fig. 4, which reports the maximum SNR $\rho_{\max }$ for the LIGO detector and for a given set of masses at a distance $d=100 \mathrm{Mpc}$. Note that the growth of $\rho_{\max }$ with $a$ becomes steeper for masses $M>200 M_{\odot}$, for which the NR-part of the waveform and hence the plunge and ringdown phase dominates. In these cases, the SNR is more then dou-

\footnotetext{
3 This behaviour can be easily understood in terms of the orbital dynamics: the binaries with larger total angular momentum will have a larger number of cycles and hence a larger SNR
}

bled between $a=-1$ and $a=+1$. Finally, when going from the present LIGO/Virgo detectors to their advanced versions, the average horizon distances go from $\sim 600 / 800 \mathrm{Mpc}$ to $\sim 10^{4} / 1.2 \times 10^{4} \mathrm{Mpc}$, thus with an observational volume of the Universe that is increased by a factor of $\sim 5000 / 3000$, respectively. Note that if we assume a Hubble radius of $\sim 4.1 \mathrm{Gpc}$, both detectors would effectively detect binaries within a large range of masses $\left(\right.$ e.g., $60 \lesssim M / M_{\odot} \lesssim 500$ for advanced LIGO) across the whole Universe.

Figure 5 shows similar information but for the planned LISA mission. Since the horizon distance can well exceed the whole Hubble horizon, the figure reports the averaged and maximum $\operatorname{SNR} \rho=\rho(a, M)$ for sources at $d=6.4 \mathrm{Gpc}(z=$ 1). Many of the considerations made above hold also for the LISA detector, and it is interesting to note that for sufficiently high and aligned spins (i.e., $a \gtrsim 0.8$ ), the SNR is $\gtrsim \mathcal{O}(10)$ already with binaries having masses $\gtrsim$ few $\times 10^{3} \widetilde{M}_{\odot}$.

Finally, the most salient information of Figs. 3 and 5 is collected in Table III which reports the properties of the "optimal" aligned binaries for the different detectors. More specifically, the Table reports in its different rows the optimal total 


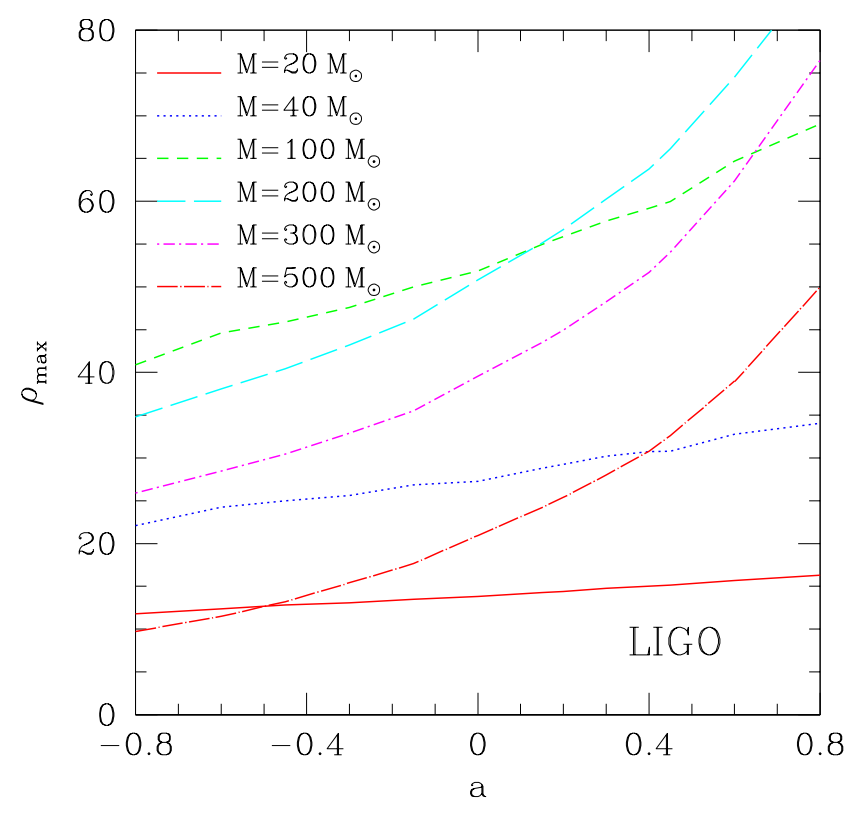

FIG. 4: Maximum SNR $\rho_{\max }=\rho(a, M)$ for the LIGO detector for a given set of masses at a distance $d=100 \mathrm{Mpc}$. Note that the growth of $\rho_{\max }$ with $a$ is very well described with a low-order polynomial which is of 4 th order for the optimal mass ( $c f$. discussion in Sect. VA . Note also that the dependence on $a$ becomes stronger for masses $M>200 M_{\odot}$, for which the NR-part of the waveform and hence the plunge and ringdown phase dominate. In these cases, the SNR is more then doubled between $a=-1$ and $a=+1$.

TABLE III: Properties of the "optimal" aligned binaries for the different detectors. Shown in the different rows are the optimal total aligned spin $a$, the optimal total mass in solar masses, the optimal maximum $\rho_{\max }$ and average $\rho_{\text {avg }}$ SNRs, the optimal horizon distance $d_{H}$ (expressed in Mpc and where $c H^{-1}$ is the Hubble radius), the lower bound for the optimal relative event rate $R$, and the glueing frequency $f_{\text {glue }}$ for the optimal binary. The masses have been sampled with an accuracy of $2.5 M_{\odot}$ for the ground-based detectors and of $2.5 \times 10^{4} M_{\odot}$ for LISA.

\begin{tabular}{|l|r|r|r|r|r|r|}
\hline \hline \hline & LIGO & eLIGO & AdLIGO & Virgo & AdVirgo & LISA \\
\hline$a$ & 0.8 & 0.8 & 0.8 & 0.8 & 0.8 & 0.8 \\
$M_{\text {opt }}\left(M_{\odot}\right)$ & 197 & 180 & 290 & 395 & 390 & $5.35 \times 10^{6}$ \\
$\rho_{\max }$ & 87 & 175 & 1667 & 118 & 1591 & $2.91 \times 10^{6}$ \\
$\rho_{\text {avg }}$ & 52 & 104 & 991 & 70 & 944 & $1.77 \times 10^{6}$ \\
$d_{H}(\mathrm{Mpc})$ & 1091 & 2190 & $>c H^{-1}$ & 1476 & $>H^{-1}$ & $>c H^{-1}$ \\
$R$ & 18 & 17 & 16 & 16 & 17 & 26 \\
$f_{\text {glue }}(\mathrm{Hz})$ & 27.48 & 30.51 & 18.71 & 13.74 & 13.91 & $1.0 \times 10^{-3}$ \\
\hline \hline
\end{tabular}

aligned spin $a$, the optimal total mass in solar masses, the optimal maximum $\rho$ and average $\rho_{\text {avg }}$ SNRs, the optimal horizon distance $d_{H}$ (expressed in Mpc and with $H^{-1}$ being the Hubble radius), the optimal relative event rate $R$, and the glueing frequency $f_{\text {glue }}$ for the optimal binary. The masses have been sampled with an accuracy of $2.5 M_{\odot}$ for the ground-based detectors and of $2.5 \times 10^{4} M_{\odot}$ for LISA.

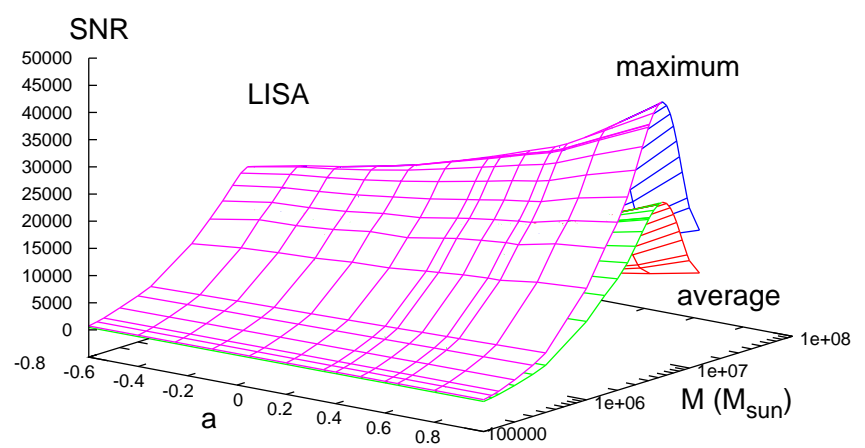

FIG. 5: Averaged and maximum SNR $\rho=\rho(a, M)$ for the planned LISA mission and for sources at $d=6.4 \mathrm{Gpc}(z=1)$.

\section{B. Influence of higher $\ell$-modes}

As discussed in Sect. IIID it is interesting to consider the impact that higher-order modes have on the SNR of equalmass aligned binaries and some representative examples of this impact is shown in Fig. 6 The left panel of this figure, in particular shows the maximum SNR $\rho_{\max }$ as a function of the mass for the highly spinning model $s_{8}$ and for the present detectors LIGO and Virgo. Different lines refer to the SNRs computed using only the $\ell=2$ multipoles (continuous line), or up to the $\ell=4$ multipoles (dashed line). Clearly, the contribution of the higher modes is most important near the optimal mass (i.e., $M \sim 200 M_{\odot}$ for LIGO and $M \sim 400 M_{\odot}$ for Virgo) but this is also non-negligible for larger masses, where it can produce an increase of $\sim 8 \%$ in SNR in a detector such as Virgo.

The right panel of Fig. 6, on the other hand, shows the ratio between maximum and averaged SNR as a function of the total projected spin $a$ for a binary of $M=200 M_{\odot}$ $\left(5.35 \times 10^{6} M_{\odot}\right)$ and the LIGO (LISA) detector. As mentioned in Sect. IIID this ratio is not expressed by a simple algebraic expression [cf. equation (9)], but needs to be determined numerically. Interestingly, this ratio is not constant but increases by $\sim 10 \%$ for larger total projected spins, underlining the importance of higher-order contributions as the initial spins increase. Overall, therefore, Fig 6 provides the answer to question (iii) in the Introduction.

\section{Match between different models}

A quantity providing a wealth of information is the match between the amplitudes of the waveforms from two different binaries, so as to quantify the differences in the gravitationalwave signal relative to some reference models. The match 

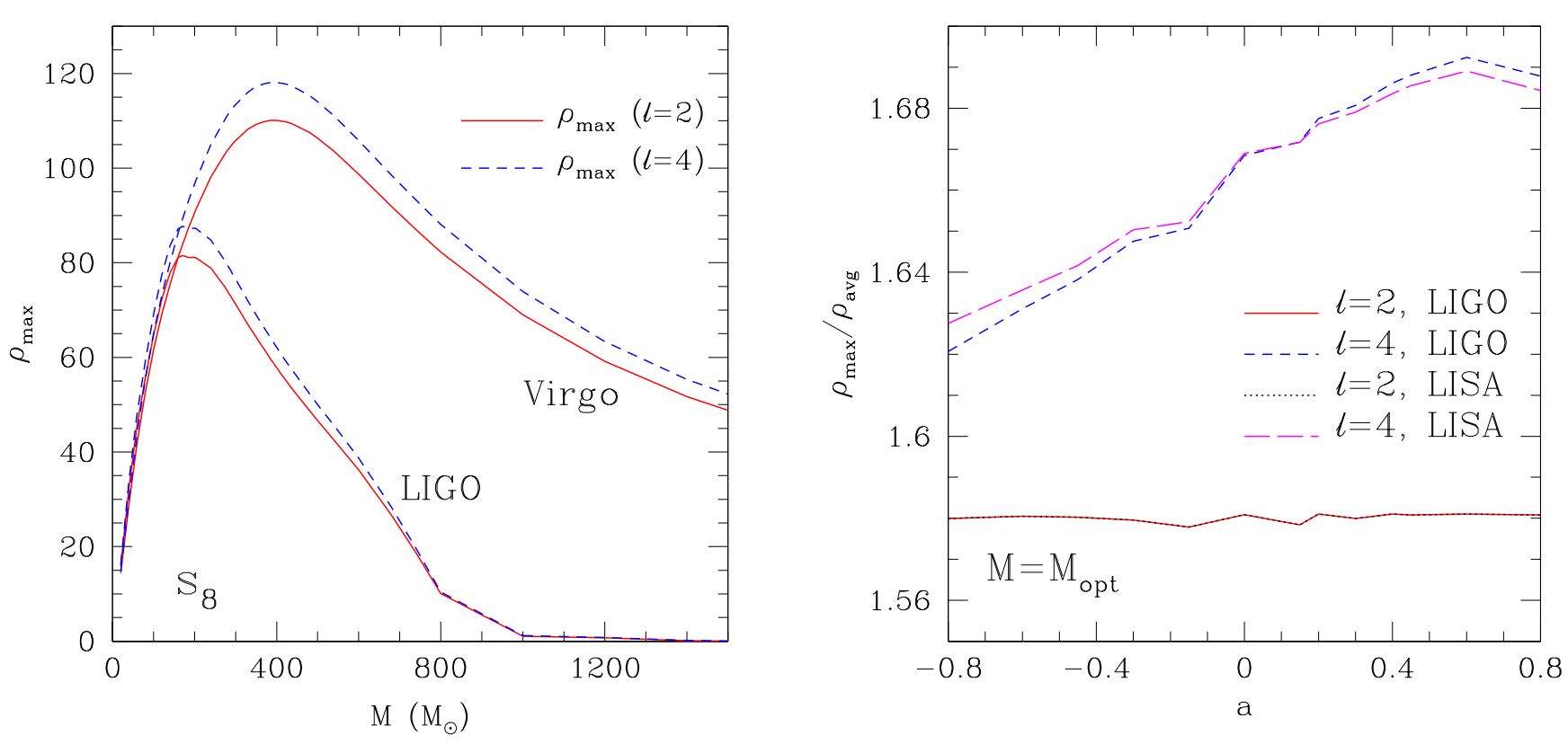

FIG. 6: Left panel: maximum SNR $\rho_{\max }$ as a function of the mass for the highly spinning model $s_{8}$ and for the present detectors LIGO and Virgo. Different lines refer to the SNRs computed using only the $\ell=2$ multipoles (continuous line), or up to the $\ell=4$ multipoles (dashed line). Right panel: ratio between maximum and averaged SNR $\rho$ as a function of the spins $a_{1}=a_{2}$ for $M=200 M_{\odot}\left(M_{-}=3.53 \times 10^{6} M_{\odot}\right)$ by including modes up to $\ell=2$ and $\ell=4$ for LIGO (LISA). In contrast to the case $\ell=2$, the $\ell=4$-curve is not constant but depends on the initial spins $a_{1}, a_{2}$

between two waveforms $h_{1}(t)$ and $h_{2}(t)$ (or a template and a waveform) can be calculated via the weighted scalar product in frequency space between two given waveforms

$$
\left\langle h_{1} \mid h_{2}\right\rangle=4 \Re \int_{0}^{\infty} d f \frac{\tilde{h}_{1}(f) \tilde{h}_{2}^{*}(f)}{S_{h}(f)},
$$

where $\tilde{h}_{1}(f)$ is the power spectral density of $h_{1}(t)$, the asterisk indicates a complex conjugate, and $S_{h}(f)$ is the noise power spectral density of a given detector. The overlap is then simply given by the normalized scalar product

$$
\mathcal{O}\left[h_{1}, h_{2}\right]=\frac{\left\langle h_{1} \mid h_{2}\right\rangle}{\sqrt{\left\langle h_{1} \mid h_{1}\right\rangle\left\langle h_{2} \mid h_{2}\right\rangle}} .
$$

Two parameters need to be taken into account when computing the overlap. The first one is the "time of arrival" $t_{\mathrm{A}}$ corresponding to an offset in the Fourier-transform of the signal exp $\left[i \omega\left(t-t_{\mathrm{A}}\right)\right]$. The second one is the "initial phase" $\Phi$ of the orbital motion when it enters the detector band.

For both of these parameters the overlap should be maximized. We have considered two possible ways of doing this. The first approach involves the best match, which gives an upper bound by maximizing over both of the phases of each waveform

$$
\mathcal{M}_{\text {best }} \equiv \max _{t_{\mathrm{A}}} \max _{\Phi_{1}} \max _{\Phi_{2}}\left\{\mathcal{O}\left[h_{1}, h_{2}\right]\right\} .
$$

The second way, instead, involves the minimax match, and is obtained by maximizing over the phase of one waveform but minimizing over the phase of the other

$$
\mathcal{M}_{\text {minimax }} \equiv \max _{t_{\mathrm{A}}} \min _{\Phi_{2}} \max _{\Phi_{1}}\left\{\mathcal{O}\left[h_{1}, h_{2}\right]\right\},
$$

and thus represents a "worst-case" scenario since it gives lower matches although one is maximizing over the template phase. More details on the maximization procedure can be found in [62, 63]. Note that all the matches computed hereafter refer to the numerical-relativity part of the waveform only.

A sensible way, if not the most sensible way, of evaluating expressions (16) and 177 is to use the binary $s_{0}$, the nonspinning binary, as a reference and to compute the overlap with the binaries at representative locations in the spin diagram, e.g., at the corners for $s_{0}-s_{8}, s_{0}-u_{8}, s_{0}-s_{-8}$, or along the main diagonal, e.g., $s_{-8}-s_{8}$. In this way we can assess whether the waveform produced by a nonspinning binary can be used to detect also spinning binaries and how much the overlap is decreased in this case.

This is shown in Fig. 7 which reports the best and minmax matches as a function of mass for a waveform containing only the $\ell=2, m=2$ contribution and refers to the LIGO detector. Different lines show the match computed between $s_{0}$ and other representative binaries, and show the remarkable similarity between the waveforms of binaries having a zero total spin. This is shown by the $s_{0}-u_{8}$ match, which is essentially very close to 1 for all the masses considered ( $c f$. also Table IV]. This result extends to all the other measured quantities, such as the radiated energy or angular momentum, and 


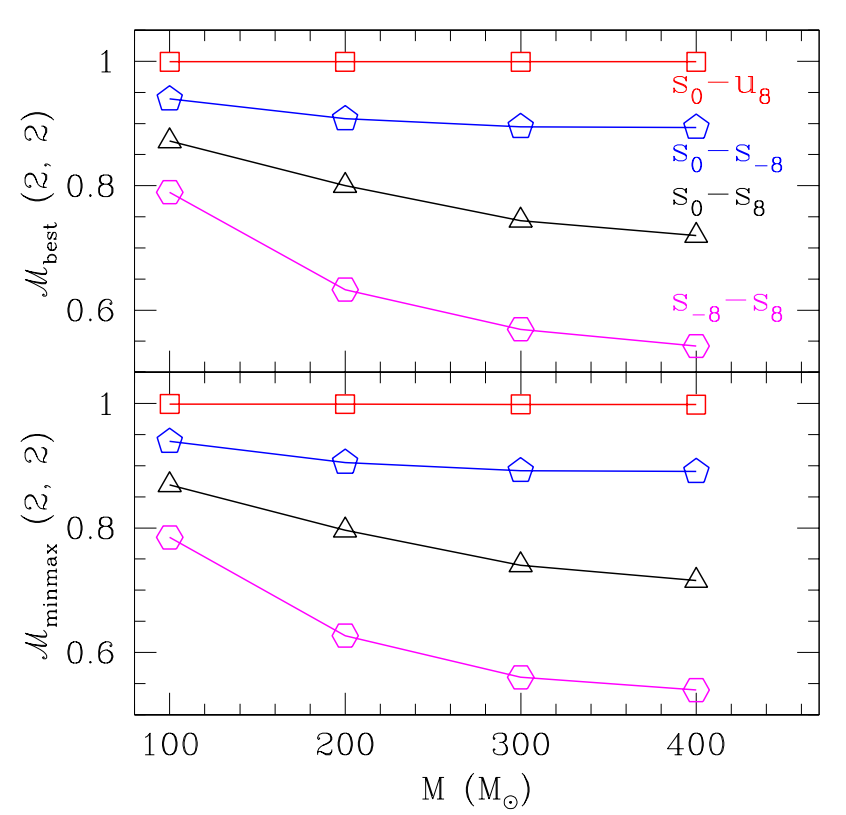

FIG. 7: Best and minmax match as a function of mass for a waveform containing only the $\ell=2, m=2$ contribution and referring to the LIGO detector. Very similar behaviors can be shown also for the other detectors.

is not particularly surprising. Indeed, it was already discussed by [63], although the investigation in that case was restricted to what is here the $u$-sequence. In addition, the equivalence between nonspinning binaries and binaries with equal and opposite spins has been exploited in the derivation of expressions for the final spin presented in a series of works [16-19]. The results of Fig. 7 and Table IV are therefore a simple example, although probably not the only possible one, of a well defined region of the space of initial configurations (i.e., those of binaries with equal masses and opposite spins) which can be mapped to an almost degenerate region (i.e., essentially to a single point) in the space of templates. This is the answer to question $(i v)$ in the Introduction and clearly represents a serious obstacle towards a proper estimate of physical parameters of the binaries that may be removed, at least in part, only if the waveform is measured with a sufficiently high SNR. A proper discussion of this problem, as well as the determination of other degenerate patches in the space of templates, will be the subject of future work.

An equally remarkable result, presented in Fig. 7 is that the overlap is also very high between the nonspinning binary and the binary with equal and antialigned spins, $s_{0}-s_{-8}$; also in this case, in fact, the best match is $\mathcal{M}_{\text {best }} \gtrsim 0.9$ for the range of masses that is relevant here. Slightly smaller and decreasing with increasing masses are the best matches computed when comparing the nonspinning binary with the binary of parallel and aligned spins, so that $\mathcal{M}_{\text {best }} \sim 0.8$, but only for very large masses. The waveforms appear clearly different (i.e., with $\mathcal{M}_{\text {best }} \lesssim 0.6$ ) only when comparing the binaries

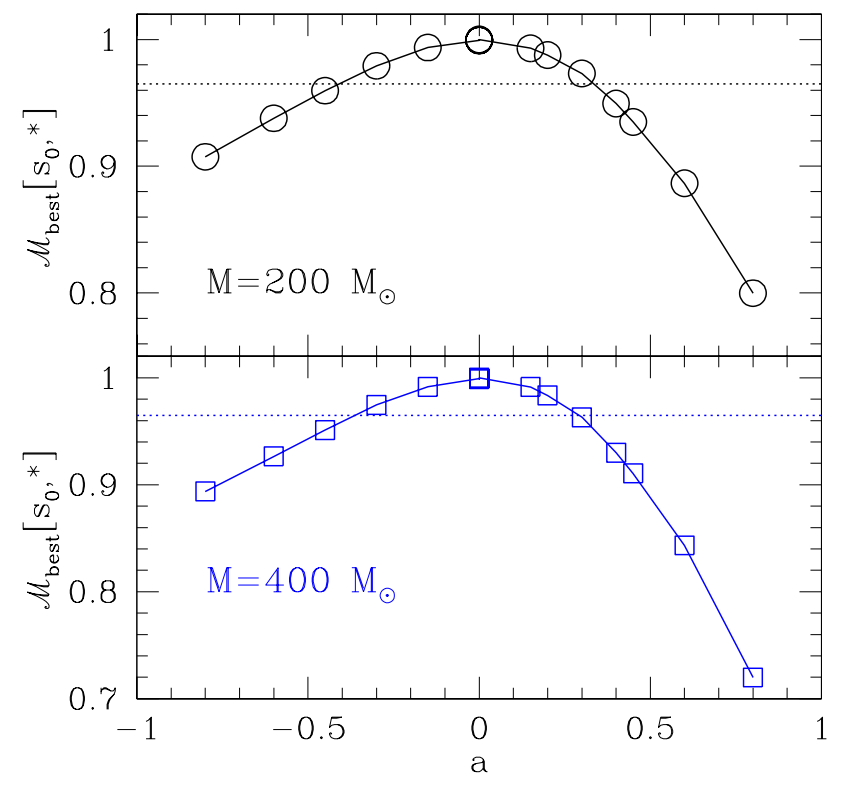

FIG. 8: Best match as a function of the total projected spin $a$ for a waveform containing only the $\ell=2, m=2$ contribution. The top/lower panels refers to a binary with a total mass $\left(200 / 400 M_{\odot}\right)$ which are close to the optimal ones for the LIGO/Virgo or advanced detectors, respectively. In both panels the dotted line shows the minimum best match $(0.965)$ needed for a detection. While the data have been computed for the LIGO detector, very similar behaviors can be shown also for the other detectors.

along the main diagonal of the spin diagram, for $s_{8}-s_{-8}$, although even in this rather extreme case the differences tend to become smaller for smaller masses. Overall, this result underlines that even simple waveforms, such as those relative to nonspinning binaries, will be effective enough to provide a detection for most configurations of equal-mass and aligned/antialigned binaries.

A different way to assess "how different" the waveforms are across all of the equal-mass aligned/antialigned spins configurations considered here is nicely summarized in Fig. 8 , which shows the best match as a function of the total projected spin $a$ for waveforms containing only the $\ell=2, m=2$ contribution and referring to the LIGO detector. The top panel, in particular, refers to a binary with a total mass of $200 M_{\odot}$ that is close to the optimal one for the LIGO/Virgo detectors, while the bottom panel refers to a binary with mass $400 M_{\odot}$ and close to the optimal one for the advanced LIGO/Virgo detectors ( $c f$. Table IV). Besides the remarkably smooth behaviour of $\mathcal{M}_{\text {best }}$ across all the values of $a$ considered, it is clear that the waveform from a nonspinning binary can be extremely useful across the whole spin diagram and yield very large overlaps even for binaries with very high spins. In both panels, in fact, the dotted line shows the minimum best match $\left(\mathcal{M}_{\text {best }}=0.965\right)$ needed for a detection [64]. This result is reassuring in light of the fact that most of the searches in the detector data are made using phenomenological waveforms 
based on nonspinning binaries.

For completeness, the results presented in Fig. 7 (as well as those in Fig. 9] are also reported in Table IV] where the different columns show $\mathcal{M}_{\text {best }}$ and $\mathcal{M}_{\text {minmax }}$ and for waveforms computed either using only the $\ell=2, m=2$ contribution (third and fourth columns), only the $\ell=3, m=2$ contribution (fifth and sixth columns), or all contributions up to $\ell=4$ (last two columns). Interestingly, the matches among the high-order modes, e.g., $\left(s_{0}\right)_{\ell=3, m=2}-\left(u_{8}\right)_{\ell=3, m=2}$, is systematically higher than those of the lower ones and remains true even for higher modes beyond $\ell=3, m=2$ which, however, we do not report here. This indicates that in order to do high-precision parameter estimation by including higher modes it is also important that these modes are accurately resolved, so that they can be clearly distinguished from one another.

We generally expect the match to degrade when the waveforms are computed by including higher-order modes (e.g., up to $\ell=4$ ) and that this degradation will become larger with increasing inclination $\theta$. The most notable example is for the degeneracy along the diagonal $a_{1}=-a_{2}$, which should be broken by the inclusion of higher-order modes (We recall that these configurations lead to different recoil velocities [16] which can only be produced by gravitational-wave contributions other than the leading order $\ell=m=2$ mode). For this reason we have computed the sky-averaged match of waveforms including modes up to $\ell=4$ (i.e., the "complete" waveforms) and the corresponding matches are reported in the last two columns of Table IV. Similarly to what was found in [63], we measure a marked decreased in the minmax match, but a much smaller decrease in the best match (the latter was not considered in [63]). Although our resolution should be marginally enough for us to detect such a difference in the best match, we also believe that a much higher accuracy is required to determine this with certainty. Note also that the matches with complete waveforms along other directions, e.g., $s_{0}-s_{8}$ or $s_{0}-s_{-8}$ do not decrease and this is simply due to the very large mismatch we already have with the $\ell=2=m$ waveforms (in these cases, in fact, the final black holes are considerably different and hence the associated ringdowns are expected to be different).

Finally, we note that although Figs 7 and 8 show data computed for the LIGO detector, very similar behaviors can be shown also for the other detectors.

\section{Accuracy of NR waveform amplitudes}

A reasonable concern that can be raised when looking the very high matches between the waveforms in the $u$-sequence is that these are simply the result of insufficient resolution. In other words, the waveforms may appear similar simply because our resolution is not sufficient to pick-up the differences. To address this concern we have computed the overlap among the waveforms obtained at three different resolutions and for a representative binary with nonzero spins, i.e., $r_{0}$. Clearly, a low match in this case would be an indication that our results are very sensitive to the numerical resolution and

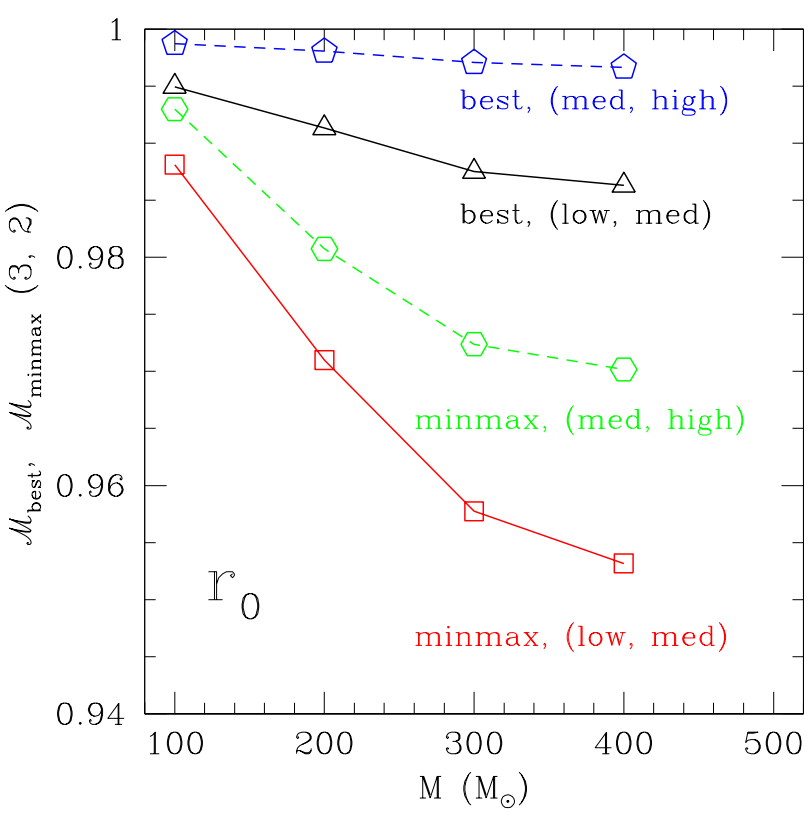

FIG. 9: As in Fig. 8 but now different lines represent the matches obtained when comparing the numerical waveforms of the binary $r_{0}$ computed at different resolutions. The matches are computed for the LIGO detector, but very similar behaviors can be shown also for the other detectors.

hence the conclusions drawn on the degeneracy of the space of templates would be incorrect.

The results of this validation are presented in Fig. 9 and are reported in the last eight rows of Table IV More specifically, shown with different lines in Fig. 9 are the matches obtained when comparing the numerical waveforms of the binary $r_{0}$ computed at low resolution $(\Delta x / M=0.024)$ and medium resolution ( $\Delta x / M=0.020$, which is also the standard one), as well as at medium and high resolution $(\Delta x / M=0.018)$. The matches are computed considering only the $\ell=2, m=2$ mode and for the LIGO detector, but very similar behaviors can be shown also for higher modes or for the other detectors.

Overall, the results reported in Fig. 9 and in Table IV show that $\mathcal{M}_{\text {best,minmax }}\left[\Delta x_{1}, \Delta x_{2}\right]>\mathcal{M}_{\text {best,minmax }}\left[h_{1}, h_{2}\right]$, i.e., that the differences we measure in the overlaps among two different waveforms $h_{1}$ and $h_{2}$ are always larger than the differences we are able to measure at two different resolutions $\Delta x_{1}$ and $\Delta x_{2}$. In other words, the differences in the waveforms across the spin diagram are always larger than our numerical errors, even along the degenerate $u$-sequence (of course, as we have a convergent numerical code, the match between medium and low resolution is worse than the match between medium and high resolution). It is also worth mentioning that as long as the dominant $\ell=2, m=2$ mode is considered, the differences in the matches are well within the margin of error for numerical-relativity simulations of black hole binaries. A recent work has in fact estimated that the differences in the waveforms produced by distinct codes is $\mathcal{M}_{\text {mismatch }}=1-\mathcal{M} \approx 10^{-4}$ for the last $\approx 1000 M$ of the 
TABLE IV: Best and minmax matches as computed for the LIGO detector for binaries with different spins in the spin diagram. Different columns show $\mathcal{M}_{\text {best }}$ and $\mathcal{M}_{\text {minmax }}$ for waveforms computed either using only the $\ell=2, m=2$ contribution (third and fourth columns), only the $\ell=3, m=2$ contribution (fifth and sixth columns), or the sky-averaged contributions of all modes up to $\ell=4$ (last two columns). Finally the last eight rows show the matches at different resolutions (i.e., $\Delta x / M=0.024,0.020,0.018$ or low, medium and high, respectively) for the binary $r_{0}$.

\begin{tabular}{|c|c|c|c|c|c|c|c|}
\hline & $M / M_{\odot}$ & $\begin{array}{c}\mathcal{M}_{\text {best }} \\
\text { only } \ell=2, m=2\end{array}$ & $\begin{array}{c}\mathcal{M}_{\operatorname{minmax}} \\
\text { only } \ell=2, m=2\end{array}$ & $\begin{array}{c}\mathcal{M}_{\text {best }} \\
\text { only } \ell=3, m=2\end{array}$ & $\begin{array}{c}\mathcal{M}_{\operatorname{minmax}} \\
\text { only } \ell=3, m=2\end{array}$ & \begin{tabular}{c|}
$\mathcal{M}_{\text {best }}$ \\
avg. up to $\ell=4$
\end{tabular} & $\begin{array}{c}\mathcal{M}_{\operatorname{minmax}} \\
\text { avg. up to } \ell=4\end{array}$ \\
\hline \multirow[t]{4}{*}{$s_{0}-s_{8}$} & 100 & 0.87182 & 0.86914 & 0.87802 & 0.85061 & 0.86337 & 0.83272 \\
\hline & 200 & 0.79987 & 0.79642 & 0.82533 & 0.80236 & 0.80070 & 0.75679 \\
\hline & 300 & 0.74394 & 0.74026 & 0.82570 & 0.78819 & 0.74785 & 0.71139 \\
\hline & 400 & 0.71981 & 0.71568 & 0.84074 & 0.81285 & 0.72345 & 0.69019 \\
\hline \multirow[t]{4}{*}{$s_{0}-u_{8}$} & 100 & 0.99926 & 0.99914 & 0.99497 & 0.97411 & 0.99673 & 0.95443 \\
\hline & 200 & 0.99928 & 0.99906 & 0.99372 & 0.95193 & 0.99483 & 0.95919 \\
\hline & 300 & 0.99923 & 0.99870 & 0.99189 & 0.93888 & 0.99251 & 0.96105 \\
\hline & 400 & 0.99919 & 0.99822 & 0.99147 & 0.93493 & 0.99110 & 0.96054 \\
\hline \multirow[t]{4}{*}{$s_{0}-s_{-8}$} & 100 & 0.93942 & 0.93907 & 0.95717 & 0.94843 & 0.93695 & 0.92143 \\
\hline & 200 & 0.90746 & 0.90536 & 0.95647 & 0.94521 & 0.89646 & 0.88041 \\
\hline & 300 & 0.89491 & 0.89197 & 0.95015 & 0.93814 & 0.87303 & 0.84960 \\
\hline & 400 & 0.89369 & 0.89065 & 0.94806 & 0.93550 & 0.85492 & 0.82103 \\
\hline \multirow[t]{4}{*}{$s_{-8}-s_{8}$} & 100 & 0.78948 & 0.78493 & 0.87041 & 0.85222 & 0.78310 & 0.74895 \\
\hline & 200 & 0.63309 & 0.62703 & 0.90722 & 0.88543 & 0.63456 & 0.59426 \\
\hline & 300 & 0.56934 & 0.56008 & 0.90322 & 0.88869 & 0.56941 & 0.52170 \\
\hline & 400 & 0.54235 & 0.53960 & 0.91199 & 0.89848 & 0.55470 & 0.49338 \\
\hline \multirow[t]{4}{*}{$s_{-8}-u_{8}$} & 100 & 0.94250 & 0.94187 & 0.96299 & 0.94669 & 0.93897 & 0.89017 \\
\hline & 200 & 0.91444 & 0.91229 & 0.96316 & 0.93068 & 0.90315 & 0.85958 \\
\hline & 300 & 0.90188 & 0.89885 & 0.95486 & 0.91256 & 0.87846 & 0.83428 \\
\hline & 400 & 0.89772 & 0.89492 & 0.95132 & 0.90583 & 0.85870 & 0.80907 \\
\hline \multirow[t]{4}{*}{$s_{8}-u_{8}$} & 100 & 0.87127 & 0.86817 & 0.87656 & 0.84229 & 0.85866 & 0.80969 \\
\hline & 200 & 0.79750 & 0.79477 & 0.83582 & 0.81476 & 0.79074 & 0.73526 \\
\hline & 300 & 0.74063 & 0.73884 & 0.83897 & 0.80378 & 0.73616 & 0.68774 \\
\hline & 400 & 0.71798 & 0.71343 & 0.84955 & 0.81925 & 0.71203 & 0.66611 \\
\hline \multirow{4}{*}{$\begin{array}{l}r_{0} \\
(0.024,0.020)\end{array}$} & $\overline{100}$ & 0.99979 & 0.99970 & 0.99495 & 0.98812 & 0.99855 & 0.99463 \\
\hline & 200 & 0.99963 & 0.99929 & 0.99133 & 0.97100 & 0.99633 & 0.98800 \\
\hline & 300 & 0.99943 & 0.99894 & 0.98752 & 0.95775 & 0.99379 & 0.98152 \\
\hline & 400 & 0.99924 & 0.99868 & 0.98630 & 0.95317 & 0.99209 & 0.97683 \\
\hline \multirow{4}{*}{$\begin{array}{l}r_{0} \\
(0.020,0.018)\end{array}$} & 100 & 0.99990 & 0.99989 & 0.99873 & 0.99299 & 0.99881 & 0.99639 \\
\hline & 200 & 0.99980 & 0.99970 & 0.99806 & 0.98074 & 0.99705 & 0.98952 \\
\hline & 300 & 0.99956 & 0.99924 & 0.99707 & 0.97238 & 0.99497 & 0.98070 \\
\hline & 400 & 0.99935 & 0.99866 & 0.99666 & 0.97017 & 0.99320 & 0.97429 \\
\hline
\end{tabular}

dominant mode of non-spinning equal mass coalescence [65]. Since the next higher mode $\ell=3, m=2$ starts to suffer from numerical noise, it does not yield the same high agreement, and the differences between best and minimax match show a larger deviation.

As a final comment on the accuracy of our waveforms, we note that the error made by using waveforms extracted at a finite radius, and not extrapolated at spatial infinity is well within the error budget of our estimates. We have validated this by comparing the waveforms extracted at a finite radius against the waveforms computed at future null infinity, via a newly developed Cauchy-characteristic code [66]. In the case of the nonspinning configuration $s_{0}$ we have found an error in the calculated SNR of less than $1.0 \%$ (details on this comparison can be found in Appendix B].

\section{FITTING FORMULAS}

In what follows we provide some simple analytic representation of most of the results presented in the previous Sections and, in particular, we give a brief discussion of fitting expressions that can be derived to express the SNR for an optimal mass and the energy radiated in gravitational waves.

\section{A. SNR}

As discussed in Sect. IIID the maximum SNR depends on several factors, most notably on the two initial spins, the total mass of the system and, although more weakly, on the number of multipoles included in the waveforms. The resulting functional dependencies when one degree of freedom is suppressed and the SNRs are presented in terms of the total projected spin are shown in Figs. 3, 5 and are clearly too cum- 


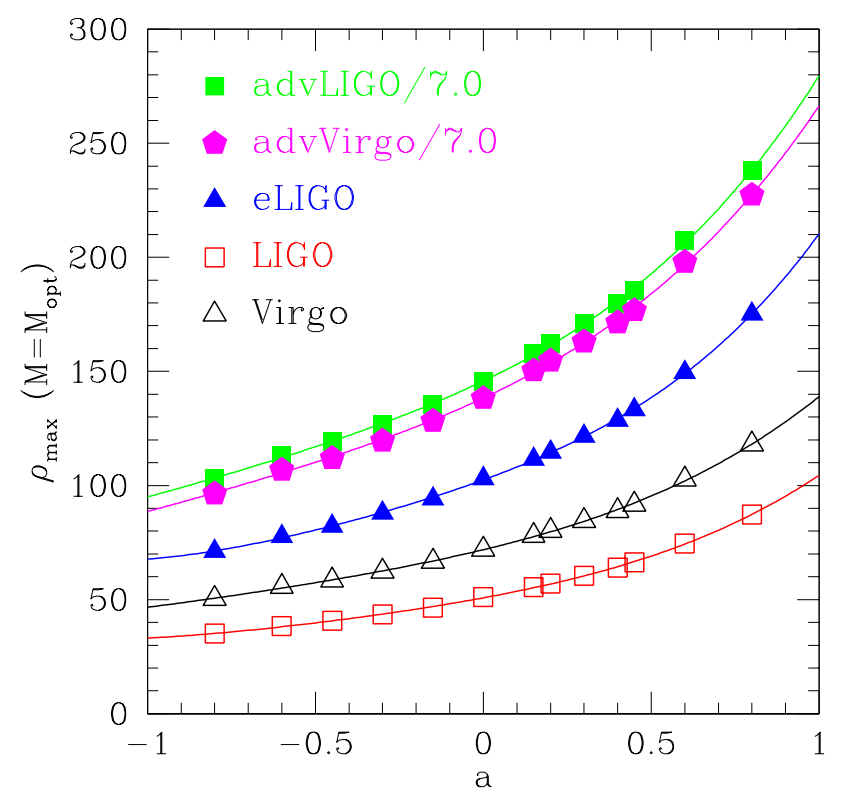

FIG. 10: Different symbols show the numerically computed values of $\rho_{\max }\left(a, M_{\mathrm{opt}}\right)$ for the different detectors and represent therefore the cross section along the optimal mass of Figs. 3 and 5 Note that the SNR for the advanced detectors have been divided by 7 to make them fit onto the same scale.

bersome to be described analytically (although still possible).

However, most of the complex functional dependence can still be captured when concentrating on the best case scenario, and hence on the SNRs relative to the optimal mass $M_{\mathrm{opt}}$. The behaviour of the SNR in this case is shown in Fig. 10, where the different symbols show the numerically computed values of $\rho_{\max }\left(a, M_{\mathrm{opt}}\right)$ for the different detectors. Stated differently, Fig. 10 represents the cross section along the optimal mass of Figs. 3] and 5 (note that the SNR for the advanced detectors have been divided by 7 to make them fit onto the same scale).

Clearly, the behaviour of the SNR in this case is sufficiently simple that it can be represented with a simple quartic polynomial of the type

$$
\rho_{\max }\left(a ; \ell \leq 4, M=M_{\mathrm{opt}}\right)=\sum_{n=0}^{4} k_{n} a^{n},
$$

whose coefficients $k_{n}$ are reported in Table $\nabla$ for the five detectors considered.

These results address therefore question (ii) formulated in the Introduction. More specifically, when considering the optimal mass, the ratio of the SNRs for maximally antialigned spinning binaries to maximally and aligned spinning binaries, i.e., $\rho_{\max }(a=1) / \rho_{\max }(a=-1)$ is $\sim 3$ for both the LIGO and Virgo detectors. This ratio is also preserved when considering the advanced LIGO and Virgo detectors. Because the event rate scales like the cube of the SNR [ $c f$. expressions (9][12] ], an increase of a factor $\sim 3$ in the SNR of binaries with
TABLE V: Fitting coefficients for the maximum SNR computed for the optimal mass [ $c f$. eq. [18] ]. The different rows refer to the various detectors and have been computed including all modes up to $\ell=4$.

\begin{tabular}{|l|r|r|r|r|r|}
\hline \hline detector & $k_{0}$ & $k_{1}$ & $k_{2}$ & $k_{3}$ & $k_{4}$ \\
\hline LIGO & 50.76 & 27.11 & 13.43 & 8.58 & 4.63 \\
LLIGO & 102.45 & 53.63 & 25.33 & 17.67 & 11.26 \\
AdLIGO & 1020.42 & 492.25 & 243.60 & 153.84 & 46.99 \\
Virgo & 71.86 & 35.23 & 17.140 & 10.92 & 3.789 \\
AdVirgo & 968.08 & 481.52 & 236.45 & 140.69 & 37.91 \\
\hline \hline
\end{tabular}

$a=-1$ and $a=1$ will translate into an increase of a factor $\sim 27$ in the event rate. It is therefore likely that many of the binaries observed will have high spins and aligned with the orbital angular momentum. This will be particularly true in the case of LISA if the prediction that the spins of supermassive black holes are aligned with the orbital angular momentum will hold [11].

\section{B. Radiated Energy}

While the SNR is effectively a measure of the amount of energy released during the inspiral, it also incorporates information on the properties of the detectors and is not therefore an absolute measure of the efficiency of the gravitational-wave emission process. This information can have a number of important astrophysical applications, and in particular it can be used to study the effect the merger has on the dynamics of the circumbinary disk accreting onto the binary when this is massive (see [67] for the first suggestion and [68] for a recent nonlinear study).

In this Section we present a simple formula to compute the amount of energy released and express it only in terms of the initial spins. Our formula is restricted to aligned binaries and is therefore not as generic as the one recently presented in [69], which however also requires the determination of a larger set of coefficients, some of which have uncertainties of $\sim 100 \%$. As we will show below, the two expressions yield results in reasonably good agreement, at least in the part of the parameter space we investigate.

In practice, the expression for the radiated energy $E_{\mathrm{rad}}$ is derived by combining a fit to the numerical data for the binaries at an initial and finite separation $D=8 M^{4}$ (we refer to this energy as to $E_{\mathrm{rad}}^{\mathrm{NR}}$ ), with the estimate of the energy released from the binary when it goes from an infinite separation down to $D$ (we refer to this energy as $E_{\mathrm{rad}}^{\mathrm{PN}}$ ), i.e.,

$$
E_{\mathrm{rad}}=E_{\mathrm{rad}}^{\mathrm{NR}}+E_{\mathrm{rad}}^{\mathrm{PN}}=M_{\mathrm{ADM}}-M_{\mathrm{fin}}+E_{\mathrm{rad}}^{\mathrm{PN}}
$$

\footnotetext{
${ }^{4}$ Note that for the binary $s_{0}$, we use an initial separation of $D=10 M$. In order to obtain the radiated energy obtained during a simulation starting from an initial separation of $D=8 M$, we only need to recalculate the initial ADM mass of the spacetime for this initial separation. The final mass of the remnant is in fact the same.
} 
where $M_{\mathrm{ADM}}$ is the initial ADM mass as measured at spatial infinity of the binary with separation $D$, and $M_{\text {fin }}$ the Christodoulou mass of the final black hole ${ }^{5}$. For the fit of the radiated energy during the numerical evolution, $E_{\mathrm{rad}}^{\mathrm{NR}}$, we use the same symmetry arguments first made in [16] and then successfully used in [17-19] to write a simple expression which is a Taylor expansion in terms of the initial spins

$\frac{E_{\mathrm{rad}}^{\mathrm{NR}}\left(q=1, a_{1}, a_{2}\right)}{M}=p_{0}+p_{1}\left(a_{1}+a_{2}\right)+p_{2}\left(a_{1}+a_{2}\right)^{2}$.

Fitting then the numerical data we obtain the following values for the coefficients

$$
\begin{aligned}
& p_{0}=\frac{3.606 \pm 0.0271}{100}, \quad p_{1}=\frac{1.493 \pm 0.0260}{100} \\
& p_{2}=\frac{0.489 \pm 0.0254}{100}
\end{aligned}
$$

where the reduced chi-squared is $\chi_{\text {red }}^{2}=0.008$, and where the largest error is in the 2nd-order coefficient but this is only $\sim 5 \%$. The different coefficients 21) can then be interpreted as the nonspinning orbital contribution to the energy loss $\left(p_{0}\right.$, which is the largest and of $\sim 3.6 \%$ ), the spin-orbit contribution ( $p_{1}$, which is $\lesssim 3.0 \%$ ), and the spin-spin contribution $\left(p_{2}\right.$, which is $\lesssim 2.0 \%$ ). The relative error between the numerically computed value of $E_{\mathrm{rad}}^{\mathrm{NR}}$ and the fitted one is reported in the last column of Table I

The PN expression for the energy radiated by the binary when going from an infinite separation down to a finite one $r=d$, depends on the total mass of the binary, the mass ratio and the spin components, i.e., $E_{\mathrm{rad}}^{\mathrm{PN}}=E_{\mathrm{rad}}^{\mathrm{PN}}\left(r, M, \nu, a_{1}, a_{2}\right)$, which is the generalization to unequal masses of the energy expression used in the definition of the TaylorT1 approximant in ref. [47]. However, exploiting the fact that for equal-mass binaries the PN radiated energy $E_{\text {rad }}^{\mathrm{PN}}$ follows the same series expansion used for $E_{\mathrm{rad}}^{\mathrm{NR}}$, we obtain for $M=1=q$

$$
\begin{aligned}
\frac{E_{\mathrm{rad}}^{\mathrm{PN}}\left(a_{1}, a_{2}\right)}{M} & =E_{\mathrm{rad}, 0}^{\mathrm{PN}} \\
& +E_{\mathrm{rad}, 1}^{\mathrm{PN}}\left(a_{1}+a_{2}\right)+E_{\mathrm{rad}, 2}^{\mathrm{PN}}\left(a_{1}+a_{2}\right)^{2},
\end{aligned}
$$

where the coefficients for $D=8 M$ are given by

$$
\begin{aligned}
& E_{\mathrm{rad}, 0}^{\mathrm{PN}}=\frac{6401}{524288} \simeq \frac{1.220}{100}, \\
& E_{\mathrm{rad}, 1}^{\mathrm{PN}}=\frac{985}{1048576 \sqrt{2}} \simeq \frac{0.0664}{100}, \\
& E_{\mathrm{rad}, 2}^{\mathrm{PN}}=-\frac{1}{32768} \simeq-\frac{0.00305}{100} .
\end{aligned}
$$

A rapid inspection of the coefficients 23 is sufficient to appreciate that the $\mathrm{PN}$ orbital contribution is only $\sim 33 \%$, the

\footnotetext{
${ }^{5}$ Note that $M_{\mathrm{ADM}}+E_{\mathrm{rad}}^{\mathrm{PN}}$ is effectively the mass of the system when it has an infinite separation. This is approximately set to 1 in most simulations but with a precision which is smaller than the one needed here.
}

one of the strong-field regime, but also that the spin-related PN contributions are mostly negligible, being at most of $\sim 4 \%$ as produced in the last orbits.

We can now combine expressions 201210 with expressions (22)-23 and estimate that for equal-mass binaries with aligned spins the energy radiated via gravitational waves from infinity is

$$
\frac{E_{\mathrm{rad}}\left(a_{1}, a_{2}\right)}{M}=\tilde{p}_{0}+\tilde{p}_{1}\left(a_{1}+a_{2}\right)+\tilde{p}_{2}\left(a_{1}+a_{2}\right)^{2},
$$

where

$$
\tilde{p}_{0}=\frac{4.826}{100}, \quad \tilde{p}_{1}=\frac{1.559}{100}, \quad \tilde{p}_{2}=\frac{0.485}{100} .
$$

Of course these numbers are specific to equal-mass binaries and refer to a situation in which the match between the PN evolution and the one in the strong-field regime is made at a specific separation of $D=8 M$. However, we expect the results to depend only weakly on this matching separation (as long as it is within a PN regime) and hence that expressions (24) and 25) are generically valid at the precision we are considering them here, namely $\sim 5 \%$.

Using expression 24 a number of quantitative considerations are possible. Firstly, the largest energy is clearly emitted by equal-mass, maximally spinning binaries with spins parallel and aligned with the orbital angular momentum at is $E_{\mathrm{rad}}(a=1) / M=9.9 \%$. Excluding the astrophysically unlikely head-on collision of two black holes moving near the speed of light (in which case $E_{\text {rad }}<14 \pm 3 \%$ [70]), these binaries are among the most efficient sources of energy in the Universe. Secondly, equal-mass nonspinning binaries lose a considerable fraction of their mass via radiation, with $E_{\mathrm{rad}}(a=0) / M=4.8 \%$, while maximally spinning binaries with spins parallel and antialigned with the orbital angular momentum have $E_{\text {rad }}(a=-1) / M=3.7 \%$.

Note that expression (24) is not a strictly monotonic function of the total spin and has a local minimum at $a_{1}=a_{2}=$ $-\tilde{p}_{1} /\left(4 \tilde{p}_{2}\right) \simeq-0.8$ rather than at $a_{1}=a_{2}=-1$, and yields $E_{\text {rad }}(a=-0.79) / M=3.6 \%$ (cf. Fig. 11). Although rather shallow, we do not expect such a local minimum. We therefore interpret it as an artifact of the numerical error of our calculations (the difference between the energy radiated at $a_{1}=a_{2}=-1$ and that at $a_{1}=a_{2}=-0.8$ is $\sim 2 \%$ and hence compatible with our overall error). Such a local minimum can be removed by adding higher-order terms in expression 201) (e.g., up to 4th order in $a_{1}+a_{2}$ ) but these improvements are so small that they do not justify the use of a more cumbersome expression. A comparison between the numerical values and the fitting expression 24 is shown in Fig. 11, where crosses and squares represent the $E_{\mathrm{rad}}^{\mathrm{NR}}$ and $E_{\mathrm{rad}}$ respectively, along the diagonal of the spin-diagram (i.e., for $a_{1}=a_{2}$ ), while the continuous line refers to our fitting expression. Note that such a line is a 1-dimensional cut of a 2-dimensional surface and hence it is not expected to exactly fit all points.

As mentioned above, Lousto and collaborators [69] have recently proposed a more general formula that should account for the radiated energy in all of the relevant space of parameters, namely for binaries with arbitrary mass ratio, spin orientation and size. Restricting their expression to the specific 


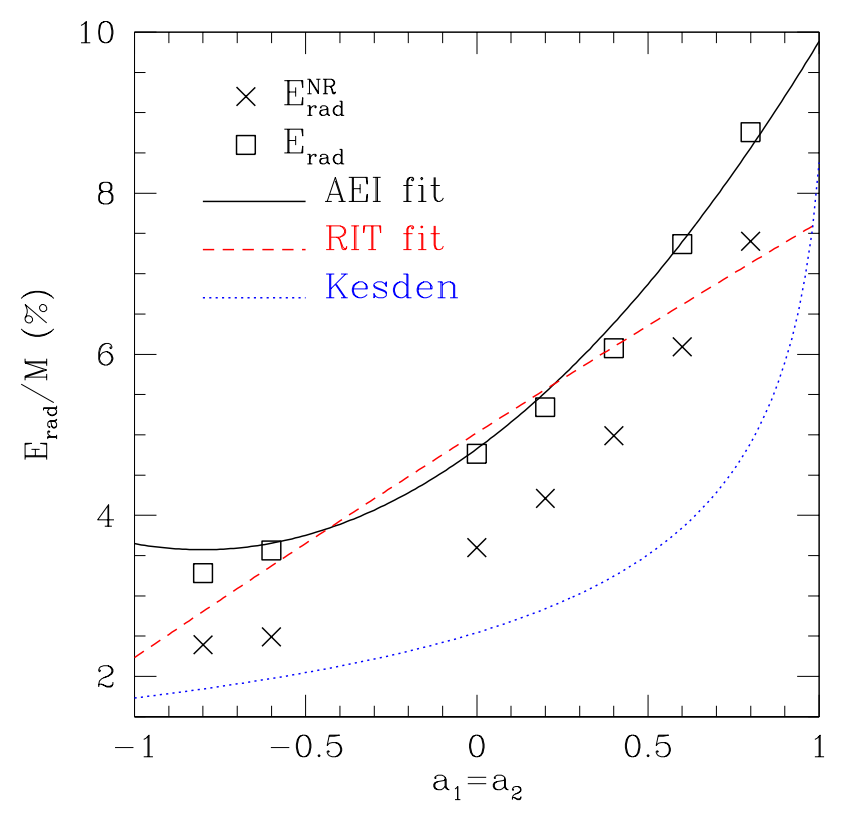

FIG. 11: Energy radiated during the numerical calculation $E_{\mathrm{rad}}^{\mathrm{NR}}$ (crosses), the total radiated energy $E_{\mathrm{rad}}=E_{\mathrm{rad}}^{\mathrm{NR}}+E_{\mathrm{rad}}^{\mathrm{PN}}$ (squares) along the diagonal of the spin diagram, i.e., for $a_{1}=a_{2}$. Shown as a continuous line is the analytic expressions given here (AEI fit), while the dashed line is the one suggested in ref. [69] (RIT fit). Note that the lines represent 1-dimensional cuts of 2-dimensional surfaces and hence are not expected to fit well all points. Finally, indicated with a dotted line is the prediction for the radiated energy coming from the point-particle approach of [20] and refined in [71].

subset of binaries considered here corresponds to setting in their expression (2): $E_{B}=E_{E}=0, \nu=1 / 4$ and $q=1$. The resulting expression is then

$$
\begin{aligned}
\frac{E_{\mathrm{rad}}^{\mathrm{RIT}}}{M} & =\frac{1}{4} E_{\mathrm{ISCO}}+\frac{1}{16} E_{2}+\frac{1}{64} E_{3} \\
& +\frac{1}{64}\left[E_{S}\left(a_{1}+a_{2}\right)+E_{A}\left(a_{1}+a_{2}\right)^{2}\right. \\
& \left.+E_{D}\left(a_{1}-a_{2}\right)^{2}\right]
\end{aligned}
$$

where the fitting coefficients have been determined to be $E_{2}=$ $0.341 \pm 0.014, E_{3}=0.522 \pm 0.062, E_{S}=0.673 \pm 0.035$, $E_{A}=-0.014 \pm 0.021, E_{D}=-0.26 \pm 0.44$ [69], and where

$$
\begin{aligned}
E_{\mathrm{ISCO}}= & \left(1-\frac{\sqrt{8}}{3}\right)+\frac{0.103803}{4} \\
& +\frac{1}{48 \sqrt{3}}\left(a_{1}+a_{2}\right)+\frac{5}{648 \sqrt{2}}\left(a_{1}-a_{2}\right)^{2} .
\end{aligned}
$$

After a bit of algebra we can rewrite 27) as

$\frac{E_{\mathrm{rad}}^{\mathrm{RIT}}\left(a_{1}, a_{2}\right)}{M}=\tilde{q}_{0}+\tilde{q}_{1}\left(a_{1}+a_{2}\right)+\tilde{q}_{2}\left(a_{1}+a_{2}\right)^{2}+\tilde{q}_{3}\left(a_{1}-a_{2}\right)^{2}$, where now

$$
\begin{aligned}
& \tilde{q}_{0}=\frac{1}{4}\left(1-\frac{\sqrt{8}}{3}+\frac{0.103803}{4}\right)+\frac{E_{2}}{16}+\frac{E_{3}}{64} \simeq \frac{5.025}{100} \\
& \tilde{q}_{1}=\frac{1}{192 \sqrt{3}}+\frac{E_{S}}{64} \simeq \frac{1.352}{100} \\
& \tilde{q}_{2}=\frac{E_{A}}{64} \simeq-\frac{0.0219}{100} \\
& \tilde{q}_{3}=\frac{5}{2592 \sqrt{2}}+\frac{E_{D}}{64} \simeq-\frac{0.270}{100}
\end{aligned}
$$

Comparing (24)-25) with (28)-(29) is now straightforward and shows that: the reduced expression from [69] has a second order contribution $\sim\left(a_{1}-a_{2}\right)^{2}$, which is absent in our expression. The remaining coefficients are rather similar but not identical. This comparison is summarized in Fig. 11, where the dashed line corresponds to the fitting proposed in ref. [69]. Note that the maximum efficiency for maximally spinning black holes predicted by expression (28) is $\sim 8 \%$, but our estimate is larger and $\sim 10 \%$. Not reported in Fig. 11 is the prediction made in ref. [72], which is linear in the total spin and very close to that coming from (28).

While the two expressions provide very similar estimates for $-0.5 \lesssim a_{1}=a_{2} \lesssim 0.4$, they also have predictions differing by more than $\sim 20 \%$ for highly spinning binaries. Because both expressions come as a result of a number of simplifications and assumptions, it is not easy to judge which one is the most accurate one, if any. It is useful to bear in mind, however, that expressions (24)-(25) have been obtained from a "controlled" set of simulations with small truncation errors and therefore have coefficients with error-bars of the order of 5\%. Expressions (28)-(29), on the other hand, because coming from more extended formulas and thus fitting a wider set of different simulations across many groups, have error-bars that are intrinsically larger, as high as $100 \%$. In view of this, and of the fact that the coefficients are constant, the simulations carried out here could be used for a new estimate of the free coefficients $E_{2}, E_{3}, E_{S}$, and $E_{A}$ in 28 (Note that because in the expression for the radiated energy (20) there is no need for a contribution proportional to $\left(a_{1}-a_{2}\right)^{2}$, it should be possible to set $\tilde{q}_{3}=0$ and obtain a numerical constraint for the presently inaccurate coefficient $E_{D}$ ). Finally, indicated with a dotted line in Fig. 11 is the prediction for the radiated energy coming from the point-particle approach of [20] and refined in [71].

Simulations involving aligned binaries with unequal masses will help to settle this issue and provide an extension to our expression (24). This will be the subject of future work.

\section{CONCLUSIONS}

We have considered in detail the issue of the detectability of binary system of black holes having equal masses and spins that are aligned with the orbital angular momentum. Because these configurations do not exhibit precession effects, they represent a natural ground to start detailed studies of the in- 
fluence of strong-field spin effects on gravitational wave observations of coalescing binaries. Furthermore, such systems are far from being unrealistic and may be the preferred endstate of the inspiral of generic supermassive binary black-hole systems. In view of this, we have computed the inspiral and merger of a large set of binary systems of equal-mass black holes with spins parallel to the orbital angular momentum but otherwise arbitrary. Our attention is particularly focused on the gravitational-wave emission so as to provide simple answers to basic questions such as what are the "loudest" and "quietest" configurations and what is the difference in SNR between the two.

Overall we find that the SNR ratio increases with the projection of the total black hole spin in the direction of the orbital momentum. In addition, equal-spin binaries with maximum spin aligned with the orbital angular momentum are more than "three times as loud" as the corresponding binaries with anti-aligned spins, thus corresponding to event rates up to 30 times larger. On average these considerations are only weakly dependent on the detectors, or on the number of harmonics considered in constructing the signal.

We have also investigated whether these binaries can lead to a degenerate patch in the space of templates. We do this by computing the mismatch between the different spinning configurations. Within our numerical accuracy we have found that binaries with opposite spins $\boldsymbol{S}_{1}=-\boldsymbol{S}_{2}$ cannot be distinguished, whereas binaries with spin $\boldsymbol{S}_{1}=\boldsymbol{S}_{2}$ have clearly distinct gravitational-wave emissions. This result, which was already discussed in the past [63], may represent a serious obstacle towards a proper estimate of the physical parameters of binaries and will probably be removed only if the SNR is sufficiently high.

Finally, we have derived a simple expression for the energy radiated in gravitational waves, and find that the binaries al- ways have efficiencies $E_{\mathrm{rad}} / M \gtrsim 3.6 \%$. This can become as large as $E_{\mathrm{rad}} / M \simeq 10 \%$ for maximally spinning binaries with spins aligned to the orbital angular momentum. These binaries are, therefore, among the most efficient sources of energy in the Universe.

\section{Acknowledgments}

It is a pleasure to thank S. Babak, E. Barausse, M. Hannam, I. Hinder, S. Hughes, B. Krishnan, L. Santamaria, B. Sathyaprakash and B. Schutz for useful discussions and comments. Mathematica codes for post-Newtonian waveforms and waveform analysis have been developed together with Mark Hannam. We thank E. Cuoco, S. Hild and M. Punturo for providing the sensitivity curve of the advanced Virgo detector. SH and DP have been supported as VESF fellows of the European Gravitational Observatory (EGO). Additional support comes from the DAAD grant D/07/13385, grant FPA-2007-60220 from the Ministerio de Educación y Ciencia (Spain), and by DFG grant SFB/Transregio 7 "Gravitational Wave Astronomy". The computations were performed at the AEI, on the LONI network (www . loni . org), at LRZ Munich, and the Teragrid (allocation TG-MCA02N014).

\section{Appendix A: Sensitivity curves}

For convenience, we report below the sensitivity curves used to compute the SNRs that are often difficult to collect from the literature. For LISA we we use the same noise curve as for the LISA Mock Data Challenge 3 [73] as implemented by Trias and Sintes, and made available by the LISA Parameter Estimation Task Force [74]. The noise curve for advanced Virgo can be found in tabulated form in Ref. [32].

LIGO

AdLIGO

$$
S_{h}(f)=S_{0}\left\{\left(\frac{4.49 f}{f_{0}}\right)^{-56}+0.16\left(\frac{f}{f_{0}}\right)^{-4.52}+0.52+0.32\left(\frac{f}{f_{0}}\right)^{2}\right\}, \quad S_{0}=9 \times 10^{-46}, \quad f_{0}=150 \mathrm{~Hz},
$$

$$
S_{h}(f)=S_{0}\left\{\left(\frac{f}{f_{0}}\right)^{-4.14}-5\left(\frac{f_{0}}{f}\right)^{2}+111\left(1-\left(\frac{f}{f_{0}}\right)^{2}+\frac{1}{2}\left(\frac{f}{f_{0}}\right)^{4}\right)\left(1+\frac{1}{2}\left(\frac{f}{f_{0}}\right)^{2}\right)^{-1}\right\}, S_{0}=10^{-49}, \quad f_{0}=215 \mathrm{~Hz}
$$

Virgo

$$
S_{h}(f)=S_{0}\left\{\left(\frac{7.87 f}{f_{0}}\right)^{-4.8}+\frac{6}{17}\left(\frac{f_{0}}{f}\right)+\left[1+\left(\frac{f}{f_{0}}\right)^{2}\right]\right\}, \quad S_{0}=10.2 \times 10^{-46}, \quad f_{0}=500 \mathrm{~Hz} .
$$

\section{Appendix B: Comparison of waveforms at future null infinity}

A systematic source of error in the results given in this paper is the finite radius $r_{\mathrm{E}}=160 \mathrm{M}$ at which our waveforms are computed. In order to determine its influence on the ac- curacy of the values reported here, we have exploited the recent possibility of computing waveforms unambiguously at future null infinity $\mathcal{J}^{+}$[66]. In this approach, which makes use of the Cauchy-characteristic extraction technique [75-79], the gravitational-wave information $\Psi_{4}$ is computed at $\mathcal{J}^{+}$in a gauge invariant way and with no causal influence from the 
outer boundary.

In practice, we have computed the match between the waveforms extracted at $r_{\mathrm{E}}$ and at $\mathcal{J}^{+}$for the nonspinning configuration $s_{0}$, and found that $\mathcal{M}_{\text {best }}=0.999$, which is thus within the error given by the match between different numerical resolutions ( $c f$. discussion in Sect. IVC and see also Table IV]. Note that the initial separation of the two black holes as reported in [66], $d=11 \mathrm{M}$, is larger than the one reported here, thus resulting in a much smaller initial frequency $\omega_{\text {ini }}$. Nevertheless, we have considered the same glueing frequency $\omega_{\text {glue }}=0.168 / M$ so as to have a fair comparison between the two waveforms.

In addition, we have also compared SNRs obtained in the two cases, when the Fourier-transform of $h(t)$ as given in terms of $\Psi_{4}$ is easily obtained as

$$
\tilde{h}(f)=-\frac{\widetilde{\Psi}_{4}}{4 \pi^{2} f^{2}},
$$

where $\widetilde{\Psi}_{4}$ is the Fourier-transform of $\Psi_{4}$. For any of the total masses considered here and for all of the detectors, we find that the differences in the SNRs is less than 1.0\%. Overall, both results show that the error introduced by the use of a finite radius calculation is within our numerical error-bars of $\sim 2.0 \%$ and thus does not modify significantly the results obtained in this work.
[1] F. Pretorius, Phys. Rev. Lett. 95, 121101 (2005).

[2] M. Campanelli, C. O. Lousto, P. Marronetti, and Y. Zlochower, Phys. Rev. Lett. 96, 111101 (2006).

[3] J. G. Baker, J. Centrella, D.-I. Choi, M. Koppitz, and J. van Meter, Phys. Rev. Lett. 96, 111102 (2006).

[4] P. Ajith et al., Class. Quant. Grav. 24, S689 (2007).

[5] P. Ajith et al., Phys. Re. D 77, 104017 (2008).

[6] P. Ajith, Class. Quant. Grav. 25, 114033 (2008).

[7] B. Aylott et al., Class. Quant. Grav. 26, 165008 (2009).

[8] B. Farr, S. Fairhurst, and B. S. Sathyaprakash, Class. Quant. Grav. 26, 114009 (2009).

[9] L. Santamaria, B. Krishnan, and J. T. Whelan, Class. Quant. Grav. 26, 114010 (2009).

[10] S. Babak, M. Hannam, S. Husa, and B. F. Schutz (2008).

[11] T. Bogdanović, C. S. Reynolds, and M. C. Miller, ApJ 661, L147 (2007).

[12] M. Koppitz, D. Pollney, C. Reisswig, L. Rezzolla, J. Thornburg, P. Diener, and E. Schnetter, Phys. Rev. Lett. 99, 041102 (2007).

[13] F. Herrmann, I. Hinder, D. M. Shoemaker, P. Laguna, and R. A. Matzner, Physical Review D 76, 084032 (2007).

[14] F. Herrmann, I. Hinder, D. Shoemaker, P. Laguna, and R. A. Matzner, Astrophys. J. 661, 430 (2007).

[15] D. Pollney, C. Reisswig, L. Rezzolla, B. Szilágyi, M. Ansorg, B. Deris, P. Diener, E. N. Dorband, M. Koppitz, A. Nagar, et al., Phys. Rev. D76, 124002 (2007).

[16] L. Rezzolla, E. N. Dorband, C. Reisswig, P. Diener, D. Pollney, E. Schnetter, and B. Szilágyi, ApJ 679, 1422 (2008).

[17] L. Rezzolla, P. Diener, E. N. Dorband, D. Pollney, C. Reisswig, E. Schnetter, and J. Seiler, ApJ 674, L29 (2008).

[18] L. Rezzolla, E. Barausse, E. N. Dorband, D. Pollney, C. Reisswig, J. Seiler, and S. Husa, Physical Review D 78, 044002 (2008).

[19] E. Barausse and L. Rezzolla, Astrophys. J. Lett. 704, L40 (2009).

[20] A. Buonanno, L. E. Kidder, and L. Lehner, Physical Review D 77, 026004 (2008).

[21] M. Campanelli, C. O. Lousto, and Y. Zlochower, Phys. Rev. D 74, 041501 (2006).

[22] M. Campanelli, C. O. Lousto, and Y. Zlochower, Phys. Rev. D 74, 084023 (2006).

[23] M. Campanelli, C. O. Lousto, and Y. Zlochower, Phys. Rev. D 73, 061501 (2006).

[24] L. Boyle, M. Kesden, and S. Nissanke, Physical Review Letters 100, 151101 (2008).
[25] L. Boyle and M. Kesden, Phys. Rev. D78, 024017 (2008).

[26] P. Marronetti, W. Tichy, B. Bruegmann, J. Gonzalez, and U. Sperhake, Phys. Rev. D77, 064010 (2008).

[27] B. Abbott et al. (LIGO Scientific Collaboration) (2007).

[28] S. Waldman and (for the LIGO Science Collaboration )., Class. Quantum Grav. 23 (2006) S653 - S660 (2006).

[29] R. Adhikari, P. Fritschel, and S. Waldman, Tech. Rep. LIGOT060156-01-I, LIGO Scientific Collaboration (2006).

[30] Advanced LIGO, http: / /www. ligo. caltech.edu/advLIGo/.

[31] F. Acernese et al., Classical Quantum Gravity 23, S635 (2006).

[32] Virgo Collaboration, advanced Virgo Preliminary Design, Virgo internal report VIR-089A-08 (2008).

[33] K. Danzmann, P. Bender, A. Brillet, I. C. A. Cruise, C. Cutler, F. Fidecaro, W. Folkner, J. Hough, P. McNamara, M. Peterseim, et al., Max-Planck-Inistitut für Quantenoptik, Report MPQ 233, 184 (1998).

[34] K. Danzmann and A. Rüdiger, Class. Quantum Grav. 20, S1 (2003).

[35] http://www. cactuscode.org.

[36] E. Schnetter, S. H. Hawley, and I. Hawke, Class. Quantum Grav. 21, 1465 (2004).

[37] J. G. Baker, J. Centrella, D.-I. Choi, M. Koppitz, and J. van Meter, Phys. Rev. D 73, 104002 (2006).

[38] M. Hannam, S. Husa, D. Pollney, B. Brugmann, and N. O’Murchadha, Phys. Rev. Lett. 99, 241102 (2007).

[39] J. Thornburg, P. Diener, D. Pollney, L. Rezzolla, E. Schnetter, E. Seidel, and R. Takahashi, Class. Quantum Grav. 24, 3911 (2007).

[40] S. Brandt and B. Brügmann, Phys. Rev. Lett. 78, 3606 (1997).

[41] J. M. Bowen and J. W. York, Phys. Rev. D 21, 2047 (1980).

[42] R. Beig and N. O. Murchadha, Class. Quant. Grav. 11, 419 (1994).

[43] S. Dain and H. Friedrich, Commun. Math. Phys. 222, 569 (2001).

[44] M. Ansorg, B. Brügmann, and W. Tichy, Phys. Rev. D 70, 064011 (2004).

[45] S. Husa, M. Hannam, J. A. Gonzalez, U. Sperhake, and B. Bruegmann, Phys. Rev. D77, 044037 (2008).

[46] A. Nagar and L. Rezzolla, Class. Quantum Grav. 22, R167 (2005), erratum-ibid. 23, 4297, (2006).

[47] M. Hannam, S. Husa, B. Bruegmann, and A. Gopakumar, Phys. Rev. D78, 104007 (2008).

[48] T. Damour, P. Jaranowski, and G. Schäfer, Phys. Lett. B 513, 147 (2001). 
[49] L. Blanchet, G. Faye, B. R. Iyer, and B. Joguet, Phys. Rev. D 65, 061501 (2002).

[50] L. Blanchet, T. Damour, G. Esposito-Farese, and B. R. Iyer, Phys. Rev. Lett. 93, 091101 (2004).

[51] L. E. Kidder, Phys. Rev. D 52, 821 (1995).

[52] E. Poisson, Phys. Rev. D57, 5287 (1998).

[53] K. Alvi, Phys. Rev. D64, 104020 (2001).

[54] L. Blanchet, A. Buonanno, and G. Faye, Phys. Rev. D74, 104034 (2006).

[55] G. Faye, L. Blanchet, and A. Buonanno, Phys. Rev. D74, 104033 (2006).

[56] M. Boyle et al., Phys. Rev. D76, 124038 (2007).

[57] L. Blanchet, G. Faye, B. R. Iyer, and S. Sinha (2008).

[58] L. E. Kidder, Phys. Rev. D 77, 044016 (2008).

[59] O. Dreyer, B. Krishnan, D. Shoemaker, and E. Schnetter, Phys. Rev. D 67, 024018 (2003).

[60] L. Baiotti, I. Hawke, P. J. Montero, F. Löffler, L. Rezzolla, N. Stergioulas, J. A. Font, and E. Seidel, Phys. Rev. D 71, 024035 (2005).

[61] E. E. Flanagan and S. Hughes, Phys. Rev. D 57, 4535 (1998).

[62] T. Damour, B. R. Iyer, and B. S. Sathyaprakash, Phys. Rev. D 57, 885 (1998).

[63] B. Vaishnav, I. Hinder, F. Herrmann, and D. Shoemaker, Phys. Rev. D76, 084020 (2007).

[64] B. Abbott et al. (LIGO), Phys. Rev. D 73, 102002 (2006).

[65] M. Hannam et al., Phys. Rev. D79, 084025 (2009).

[66] C. Reisswig, N. T. Bishop, D. Pollney, and B. Szilagyi, Phys.
Rev. Lett. 103, 221101 (2009).

[67] N. Bode and S. Phinney, APS Meeting Abstracts pp. 1010-+ (2007).

[68] M. Megevand, M. Anderson, J. Frank, E. W. Hirschmann, L. Lehner, S. L. Liebling, P. M. Motl, and D. Neilsen, Phys. Rev. D. 80, 024012 (2009).

[69] C. O. Lousto, M. Campanelli, and Y. Zlochower (2009).

[70] U. Sperhake, V. Cardoso, F. Pretorius, E. Berti, and J. A. Gonzalez, Phys. Rev. Lett. 101, 161101 (2008).

[71] M. Kesden, Physical Review D 78, 084030 (2008).

[72] W. Tichy and P. Marronetti, Physical Review D 78, 081501 (2008).

[73] S. Babak et al., Class. Quant. Grav. 25, 184026 (2008).

[74] LISA parameter estimation wiki, http: //www.tapir.caltech.edu/dokuwiki/lisape: home.

[75] N. T. Bishop, Class. Quantum Grav. 10, 333 (1993).

[76] N. Bishop, R. Isaacson, R. Gómez, L. Lehner, B. Szilágyi, and J. Winicour, in Black Holes, Gravitational Radiation and the Universe, edited by B. Iyer and B. Bhawal (Kluwer, Dordrecht, The Neterlands, 1999), p. 393.

[77] M. Babiuc, B. Szilágyi, I. Hawke, and Y. Zlochower, Class. Quantum Grav. 22, 5089 (2005).

[78] M. C. Babiuc, N. T. Bishop, B. Szilágyi, and J. Winicour, Phys. Rev. D79, 084011 (2009).

[79] N. T. Bishop, R. Gómez, L. Lehner, and J. Winicour, Phys. Rev. D 54, 6153 (1996). 